\title{
Isolated ionospheric disturbances as deduced from global GPS network
}

\author{
E. L. Afraimovich, E. I. Astafieva, and S. V. Voyeikov \\ Institute of Solar-Terrestrial Physics SD RAS, P. O. box 4026, Irkutsk, 664033, Russia \\ Received: 9 December 2002 - Revised: 10 June 2003 - Accepted: 18 June 2003 - Published: 1 January 2004
}

\begin{abstract}
We investigate an unusual class of medium-scale traveling ionospheric disturbances of the nonwave type, isolated ionospheric disturbances (IIDs) that manifest themselves in total electron content (TEC) variations in the form of single aperiodic negative TEC disturbances of a duration of about $10 \mathrm{~min}$ (the total electron content spikes, TECS). The data were obtained using the technology of global detection of ionospheric disturbances using measurements of TEC variations from a global network of receivers of the GPS. For the first time, we present the TECS morphology for 170 days in 1998-2001. The total number of TEC series, with a duration of each series of about $2.3 \mathrm{~h}(2 \mathrm{~h} 18 \mathrm{~m})$, exceeded 850000 . It was found that TECS are observed in no more than $1-2 \%$ of the total number of TEC series mainly in the nighttime in the spring and autumn periods. The TECS amplitude exceeds the mean value of the "background" TEC variation amplitude by a factor of 5-10 as a minimum. TECS represent a local phenomenon with a typical radius of spatial correlation not larger than $500 \mathrm{~km}$. The IID-induced TEC variations are similar in their amplitude, form and duration to the TEC response to shock-acoustic waves (SAW) generated during rocket launchings and earthquakes. However, the IID propagation velocity is less than the SAW velocity (800$1000 \mathrm{~m} / \mathrm{s}$ ) and are most likely to correspond to the velocity of background medium-scale acoustic-gravity waves, on the order of $100-200 \mathrm{~m} / \mathrm{s}$.
\end{abstract}

Key words. Ionosphere (ionospheric irregularities, instruments and techniques) - Radio science (ionospheric propagation)

\section{Introduction}

Of the known ionospheric irregularities of a different class, mid-latitude isolated ionospheric disturbances (IIDs) stand out as a highly unusual type. There has been a consistent interest over the past 40 years in the study of the origin of

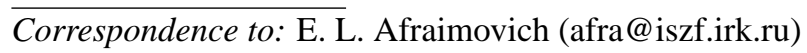

IIDs that was aroused due to difficulties in determining an adequate mechanism for IID generation in mid-latitudes, as well as by the fact that the IID have a marked effect on amplitude and phase characteristics of transionospheric signals from radio engineering communication and navigation systems (Titheridge, 1971; Karasawa, 1985; Bowman, 1989; Afraimovich et al., 1992) by causing serious malfunctions of these systems.

A large number of publications (e.g. Karasawa, 1985; Titheridge, 1971), including a review by Bowman (1989) were devoted to the study along this line. IIDs are detected when recording amplitude and phase scintillations of transionospheric radio signals in the form of rarely occurring single aperiodic negative impulses with a duration from a few to several tens of seconds (Karasawa, 1985). To name this uncommon type of scintillation Karasawa (1985) seems to be the first to coin the term "spikes-type" (S-) scintillations.

Karasawa et al. (1985) noticed from a long-term recording of the signal from the geostationary MARISAT satellite at $1.5 \mathrm{GHz}$ frequency which was synchronous with amplitude S-scintillations, there occur similar-appearing changes to the rotation angle of the polarization plane that are proportional to a corresponding disturbance of total electron content (TEC). Anomalous fluctuations, recorded during 13 months of observation, occur predominantly in the nighttime and last from $5 \mathrm{~s}$ to $2 \mathrm{~min}$. The diurnal dependence of the S-type oscillations shows two distinct peaks: 09:00-15:00 in the daytime, and 20:00-01:00 at night. As far as the seasonal dependence is concerned, however, a maximum of the oscillation distribution corresponds to early summer (June). It is customary to associate the occurrence of S-type oscillations with diffraction or interference from small-scale irregularities, "blobs" and "bubbles", generated in the ionosphere.

Titheridge (1971) found that amplitude and phase S-scintillations are caused by refraction and diffraction effects at the propagation of the transionospheric signal in a medium with IIDs and presented the corresponding formulae for estimating these effects as a function of the relationship of the wavelength of the radio wave, the irregularity size and 
the sounding geometry (the distance to the satellite and to the layer with IIDs).

However, in spite of the many years of experimental and theoretical investigations, there is as yet no clear understanding not only of the physical nature of IIDs, but even of their morphology (the occurrence frequency as a function of geographical position, time, level of geomagnetic and meteorological activity, etc.).

To tackle these questions requires statistically significant sets of experimental data with good spatial and temporal resolution, in order to gain insight into not only morphological, but also dynamic IID characteristics: the direction of travel, the propagation velocity, and the location of the possible disturbance source. Another important requirement implies a continuity and global coverage of observations, since such phenomena are relatively rare in time and random in space.

Such an opportunity is for the first time provided by the use of the international ground-based network of two-frequency receivers of the GPS, consisting of no less than 1000 sites as of the beginning of 2002 and posting its data on the Internet, which opens up new avenues for a global, continuous, fully computerized monitoring of ionospheric disturbances of a different class.

The objective of this paper is to study the morphology and spatial and temporal properties of IIDs using data from a global network of GPS receivers. Following Karasawa et al. (1985), the term TECS (total electron content spikes) will be used here to designate the IID-induced TEC disturbances. The sample statistic of the occurrence frequency and morphology used in this study does refer to TECS recorded from GPS data. Within the framework of certain model representations, using these data it is possible to reconstruct amplitude and spatial characteristics of local electron density disturbances, i.e. of IIDs themselves. On this basis, the term IIDs will be used below interchangeably with the term TECS.

Section 2 describes the method for detecting the TECS obtained in our study. Section 3 presents the TECS morphology. Section 4 is devoted to a detailed analysis of the spatial and temporal properties of IIDs by considering the most pronounced manifestation of TECS on 5 October 2001 in California, USA. The discussion of results compared with findings reported by other authors is presented in Sect. 5 .

Our comparison of IID characteristics with geomagnetic field variations used data from near-lying magnetic variation stations of the INTERMAGNET network (address: http: //www.intermagnet.org).

\section{Method of processing the data from the global net- work: selection of TECS}

The standard GPS technology provides a means for wave disturbance detection based on phase measurements of TEC at each of the spaced two-frequency GPS receivers (HofmannWellenhof et al., 1992):

$$
I_{o}=\frac{1}{40.308} \frac{f_{1}^{2} f_{2}^{2}}{f_{1}^{2}-f_{2}^{2}}\left[\left(L_{1} \lambda_{1}-L_{2} \lambda_{2}\right)+\text { const }+n L\right],
$$

where $L_{1} \lambda_{1}$ and $L_{2} \lambda_{2}$ are additional paths of the radio signal caused by the phase delay in the ionosphere, $(\mathrm{m}) ; L_{1}$ and $L_{2}$ represent the number of phase rotations at the frequencies $f_{1}$ and $f_{2} ; \lambda_{1}$ and $\lambda_{2}$ stand for the corresponding wavelengths, (m); const is the unknown initial phase ambiguity, (m); and $n L$ are errors in determining the phase path, (m).

Phase measurements in the GPS can be made with a high degree of accuracy corresponding to the error of TEC determination of at least $10^{14} \mathrm{~m}^{-2}$ when averaged on a 30 s time interval, with some uncertainty of the initial value of TEC, however (Hofmann-Wellenhof et al., 1992). This makes it possible to detect ionization irregularities and wave processes in the ionosphere over a wide range of amplitudes (up to $10^{-4}$ of the diurnal TEC variation) and periods (from $24 \mathrm{~h}$ to $5 \mathrm{~min}$ ). The unit of TEC, TECU, which is equal to $10^{16} \mathrm{~m}^{-2}$, is commonly accepted in literature, and will be used in the following text.

Primary data include a series of "oblique" values of TEC $I_{0}(t)$, as well as the corresponding series of elevations $\theta_{S}(t)$ and azimuths $\alpha_{s}(t)$ of the Line-of-Sight (LOS) to the satellite, calculated using our developed program which converts the GPS system standard RINEX-files on the Internet (Gurtner, 1993).

A series of the values of elevations $\theta_{S}(t)$ and azimuths $\alpha_{s}(t)$ of the LOS to the satellite were used to determine the coordinates of subionospheric points for the height $h_{\max }=300 \mathrm{~km}$ of the $F_{2}$-layer maximum, and to convert the "oblique" TEC $I_{o}(t)$ to the corresponding value of the "vertical" TEC by employing the technique reported by Klobuchar (1986)

$I=I_{o} \times \cos \left[\arcsin \left(\frac{R_{z}}{R_{z}+h_{\max }} \cos \theta_{s}\right)\right]$,

where $R_{z}$ is the Earth's radius. All results in this study were obtained for elevations $\theta_{S}(t)$ larger than $30^{\circ}$.

To eliminate variations of the regular ionosphere, as well as trends introduced by orbital motion of the satellite, we obtained TEC variations $d I(t)$ by filtering from the initial $I(t)$-series over the range of periods of 2-20 min.

The technology of global detection of TEC disturbances of a natural and technogenic origin that was developed at the ISTP SB RAS (Afraimovich, 2000b) makes it possible to select - in the automatic mode from an extensive amount of experimental material - TEC variations which can be classed as TECS.

TECS were selected by two criteria. TEC variations were selected first, where the standard deviation (rms) exceeded the prescribed level $\epsilon$. The statistic of TECS discussed in this paper was obtained for $\epsilon=0.1 T E C U$. Our selection of the threshold value of $\epsilon$ is based on the following considerations. Afraimovich et al. (2001a) investigated the spectra of ionospheric TEC disturbances for quiet and disturbed geomagnetic conditions using GPS data and showed that the rms of the TEC variation over the range of periods of 2-20 min for quiet geomagnetic conditions is $0.005 T E C U$. It is this range that corresponds to the typical TECS periods. During geomagnetic disturbances, the TEC variation amplitude 


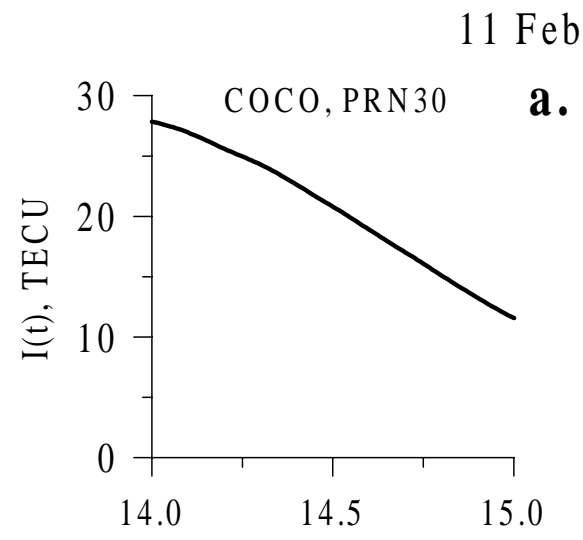

uary 2001
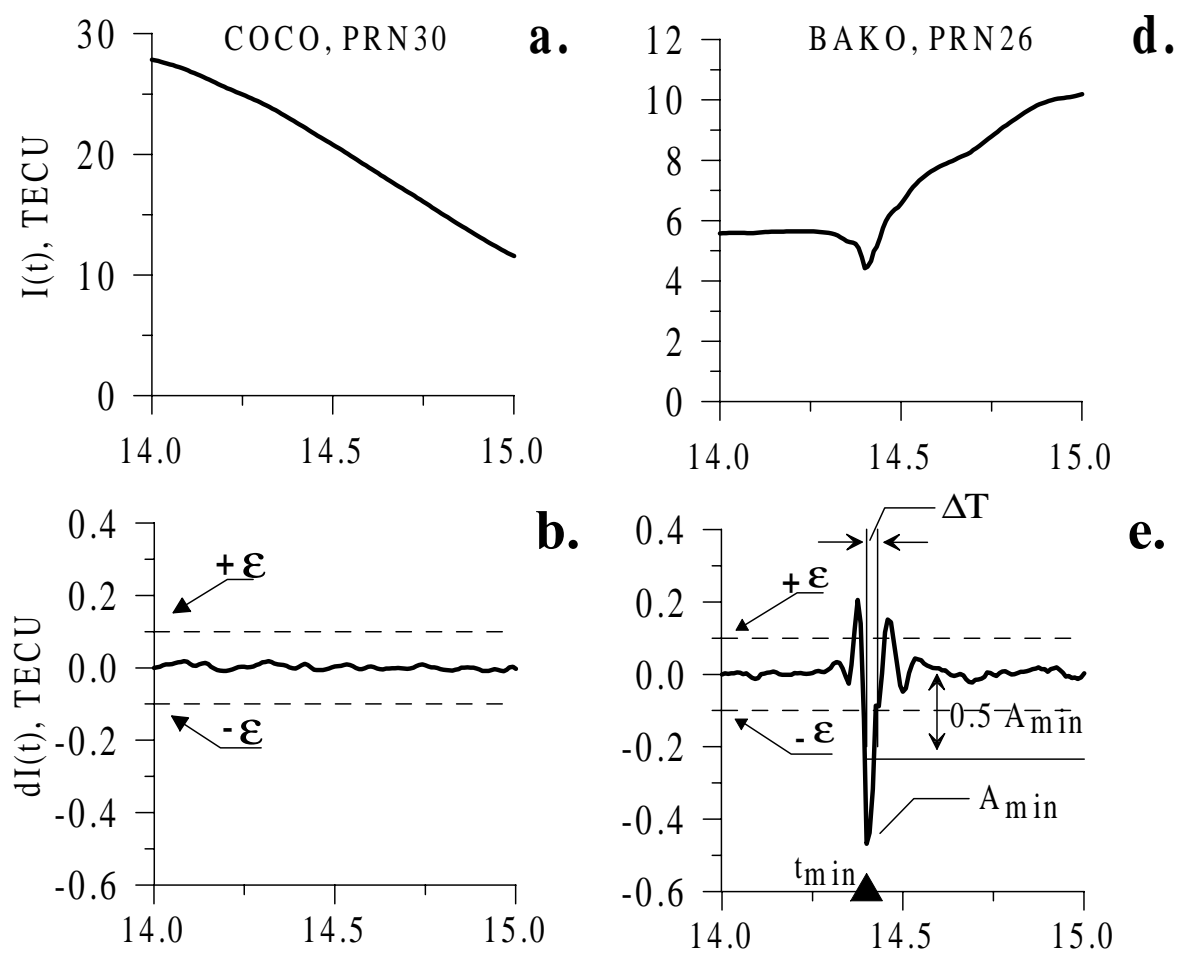

5 October 2001
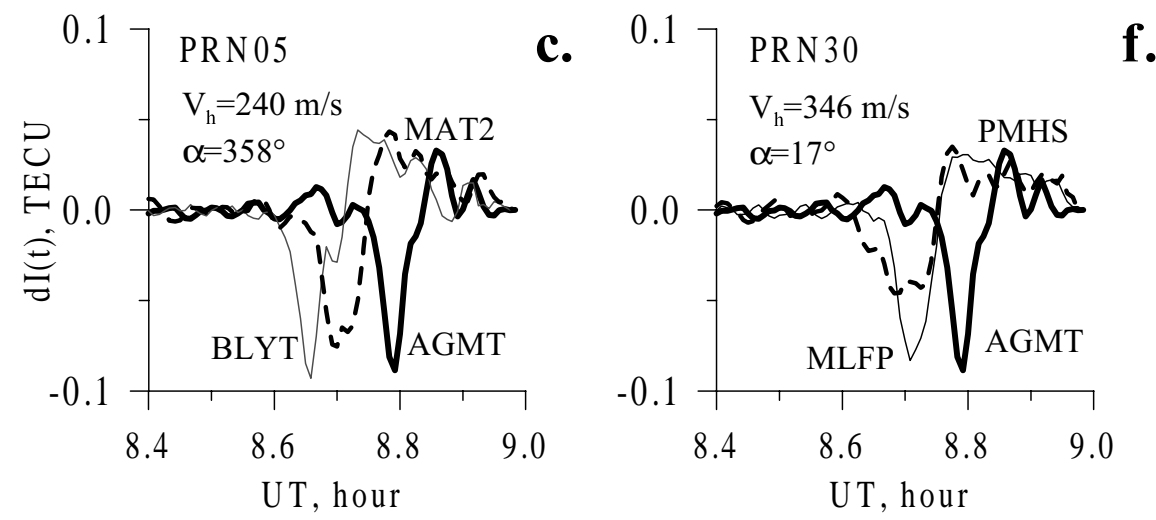

Fig. 1. An illustration of TECS selection: (a) - an example of a typical "vertical" TEC $I(t)$ series (containing no TECS) recorded on 11 February 2001 at station COCO $\left(96.8^{\circ} \mathrm{E} ; 12.1^{\circ} \mathrm{S}\right)$; (b) - filtered $d I(t)$-variations (from the initial $I(t)$-series). Panels (d) and (e) - same but for station BAKO $\left(106.8^{\circ} \mathrm{E} ; 06.5^{\circ} \mathrm{S}\right)$, the $I(t)$-series containing TECS. Shown in panels (a) and (d) are the station names and GPS satellite numbers (PRN). Panel (e) shows the minimum value of the amplitude $A_{\min }$, the time $t_{\min }$ corresponding to this amplitude, and the time duration $\Delta T$ is shown in (e) by the shaded triangle. Panels (c) and (f) (for PRN05 and PRN30, respectively) present the filtered TECS for spaced GPS stations on 5 October 2001.

can increase an order of magnitude compared to a magnetically quiet period and reach $0.05 T E C U$. Thus, the value 0.1 TECU permits filtering of the background TEC fluctuations.

The aforementioned estimates of the typical values of rms of $d I(t)$ variations were obtained by Afraimovich et al. (2001a) by a global averaging of the data from the GPS network and represent daily mean values. In Afraimovich et al. (2001a) it was pointed out that rms of $d I(t)$ variations for the daytime conditions generally exceeded the respective nighttime values no more than by a factor of 2-4. For that reason, by selecting of the threshold value $\epsilon=0.1$, it was possible to eliminate the background TEC fluctuations for both nighttime and daytime conditions.

Figure 1 illustrates the TECS selection procedure. Figure 1a presents an example of weakly disturbed disturbances of a "vertical" TEC $I(t)$ recorded on 11 February 2001 at station COCO. Figure $1 \mathrm{~b}$ presents the filtered $d I(t)$-variations. 

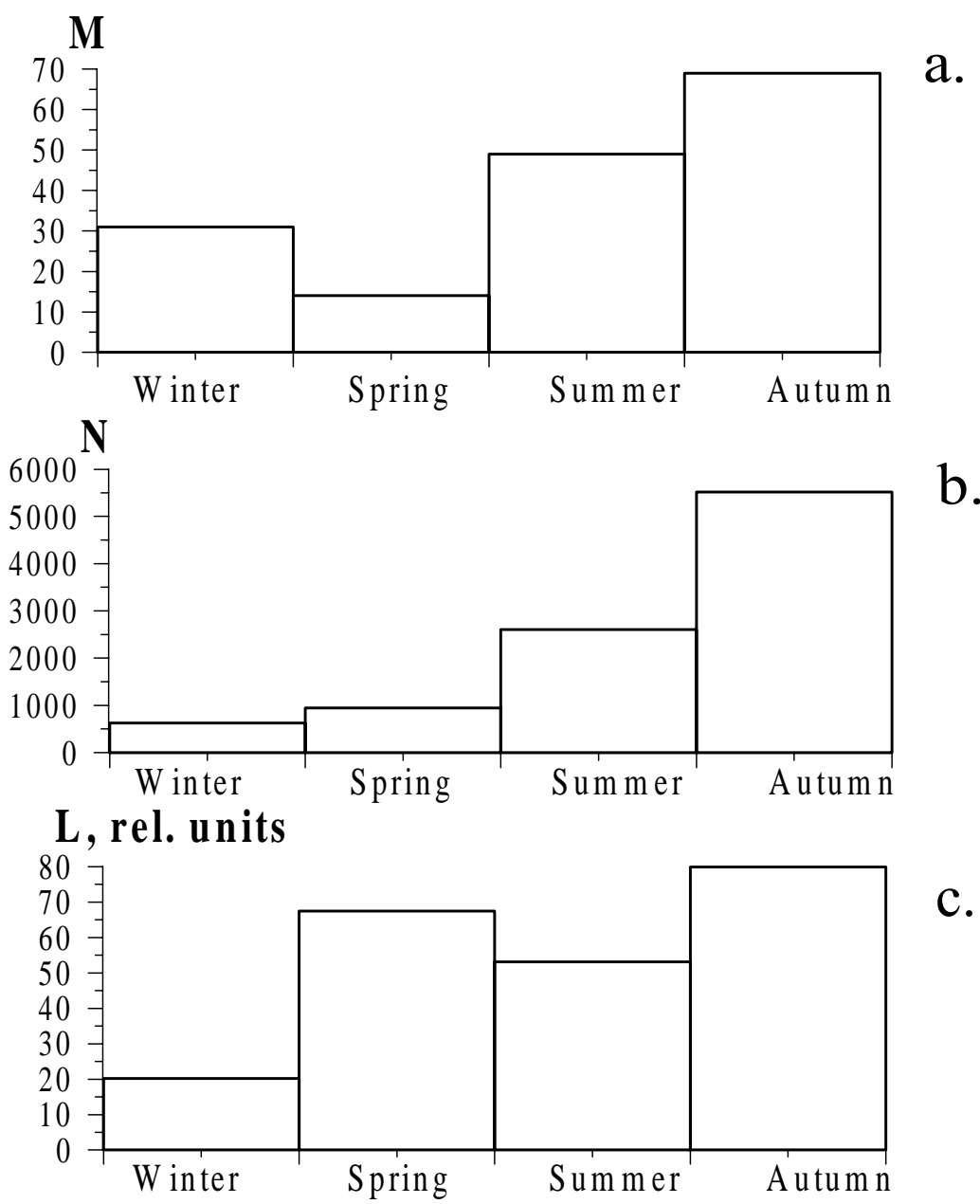

c.

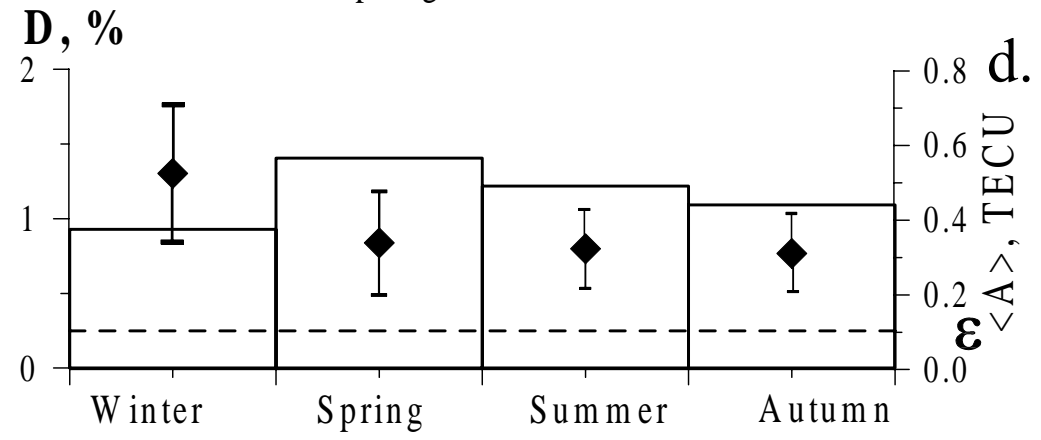

Fig. 2. Seasonal dependence of the density and maximum amplitude of TECS: (a) - number of days $M$ of observation versus time of the year; (b) - number of TECS $N$; (c) - mean number of TECS per day $L=N / M$. (d) - relative TECS density $D$ obtained as the ratio of the number of TECS $N$ to the number of receiver-satellite Lines-of-Sight. Diamonds in panel (d) show the mean values of $\langle A\rangle$ of the maximum amplitudes $\left|A_{\min }\right|$ for each season, and vertical lines show their rms. The dashed horizontal line shows the threshold in amplitude $(\epsilon=0.1 T E C U)$

Dashed horizontal lines show the prescribed threshold $\epsilon$. The rms of the $d I(t)$-variations is $0.007 T E C U$, that is, does not reach the prescribed threshold, $\epsilon=0.1 T E C U$.

Figures 1d and e plot the same dependencies as in Figs. 1a and $b$, but for station BAKO. It is evident from Fig. 1d that unusual (for background disturbances) TEC variations in the form of a single impulse of a duration on the order of $\Delta T=$ 10 min are clearly identified at the background of slow TEC variations.
The time $t_{\min }$, corresponding to the value of $A_{\min }$ of the filtered TEC variation $d I(t)$, is shown in Fig. 1e by the shaded triangle. The value of $\Delta T$ is determined from the level of $0.5 A_{\text {min }}$. The amplitude of this pulse $A_{\min }$ far exceeds the specified threshold $\epsilon=0.1 T E C U$ and is at least an order of magnitude higher than the background TEC fluctuation intensity for this range of periods (Afraimovich et al., 2001a).

Then for each filtered series we verified the fulfilment of the "singleness" condition of the TEC overshoot. The condi- 
tion of uniqueness of the TECS impulse on the time interval of $2.3 \mathrm{~h}$ used in the analysis was satisfied if the maximum value of $\left|A_{\min }\right|$ exceeded the rms value of $d I(t)$ at least by a factor of 3 and the amplitude of a secondary minimum of $d I(t)$ did not exceed $0.5 A_{\min }$ (see Fig. 1e). Furthermore, we discarded the TEC impulses whose duration $\Delta T$ exceeded the specified value $30 \mathrm{~min}$.

It should be noted that the above examples (Figs. 1b and e) both refer to the same time interval and to the stations spaced by a distance over $1300 \mathrm{~km}$. This indicates the local character of the phenomenon and is in agreement with the overall statistic characterizing its spatial correlation (see Sect. 3).

For each of the events satisfying the above TECS selection criteria, a special file was used to store information about the GPS station name and geographic latitude and longitude; GPS satellite PRN number; amplitude $A_{\text {min }}$; time $t_{\text {min }}$ corresponding to the minimum value of the $A_{\min }$; and about the TECS duration $\Delta T$. The sample statistic, presented below, was obtained by processing such files.

\section{Morphology of TECS}

The total number of the TEC series for 170 days in 19982001 , used in the analysis, corresponding to the observation along a single receiver-satellite LOS, with a duration of each series of about $2.3 \mathrm{~h}$, exceeded 850000 . The method outlined above was used to obtain a set of TECS totaling about 10000 cases, or making up about $1 \%$ of the total number of the Lines-of-Sight. An analysis of the resulting statistic revealed a number of dependencies of TECS parameters on different factors.

First, we consider the seasonal dependence of the TECS' occurrence frequency and amplitude (Fig. 2). Figure 2a plots the number of days of observation $M$ versus season. As is evident, autumn is represented best statistically. Figure $2 b$ shows the seasonal dependence of the number of TECS, $N$. Figure 2c plots the number of TECS per day as a function of season $L=N / M$. This dependence has maxima in spring and in autumn.

The relative density of TECS, $D$, obtained as the ratio of the number of TECS identified, $N$, to the number of Linesof-Sight, is presented in Fig. 2d. Vertical lines in Fig. 2d show the TECS standard deviations (rms). The most probable value of $\left\langle\left|A_{\min }\right|\right\rangle$ with a small scatter varies around the value $0.3 T E C U$, independently of the season.

Figure $3 \mathrm{a}$ presents the diurnal distribution of TECS, $P\left(t_{\min }\right)$, for the times $t_{\min }$. It is evident that the distribution has maxima in the nighttime and in the morning hours, approximately from 00:00 to 07:00, and from 23:00 to 24:00 of local time LT.

Figure $3 \mathrm{c}$ presents the normalized probability distribution $P\left(\left|A_{\min }\right|\right)$ of TECS occurrence with a given amplitude $\left|A_{\min }\right|$. It was found that the largest probable value of the amplitude $\left|A_{\min }\right|$, also shown in Fig. $3 \mathrm{c}$, is about $0.3 \mathrm{TECU}$, and the half-width of the distribution is 0.2 TECU. Thus, the most probable value of the amplitude $\left|A_{\min }\right|$ of the TECS
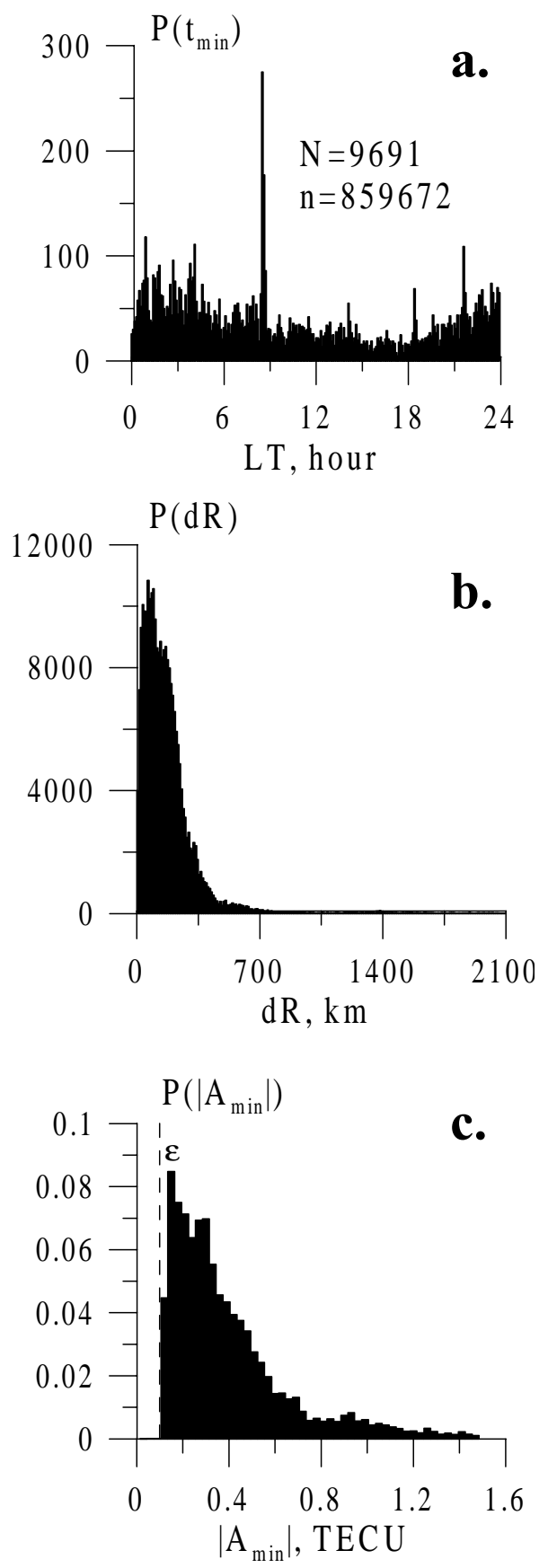

Fig. 3. Statistics of TECS: (a) - LT diurnal distribution $P\left(t_{\min }\right)$ of the times $t_{\min }$ corresponding to the minimum amplitude $A_{\min }$ of the TECS; (b) - histogram $P(d R)$ of the number of cases where TECS within one $2.3 \mathrm{~h}$ time interval were observed at any two GPS stations, with the distance $d R$ between them; (c) - distribution $P\left(\left|A_{\min }\right|\right)$ of the maximum amplitude $\left|A_{\min }\right|$ of TECS. The vertical dashed line in panel (c) shows the threshold in amplitude $\epsilon=0.1 T E C U$. Panel (a) shows the number $N$ of the detected TECS, and the total number $n$ of Lines-of-Sight

identified here exceeds the mean value of the "background" TEC variation amplitude by a factor of 5-10 as a minimum (Afraimovich et al., 2001a). 
5 October 2001

08:00 - 10:00 UT
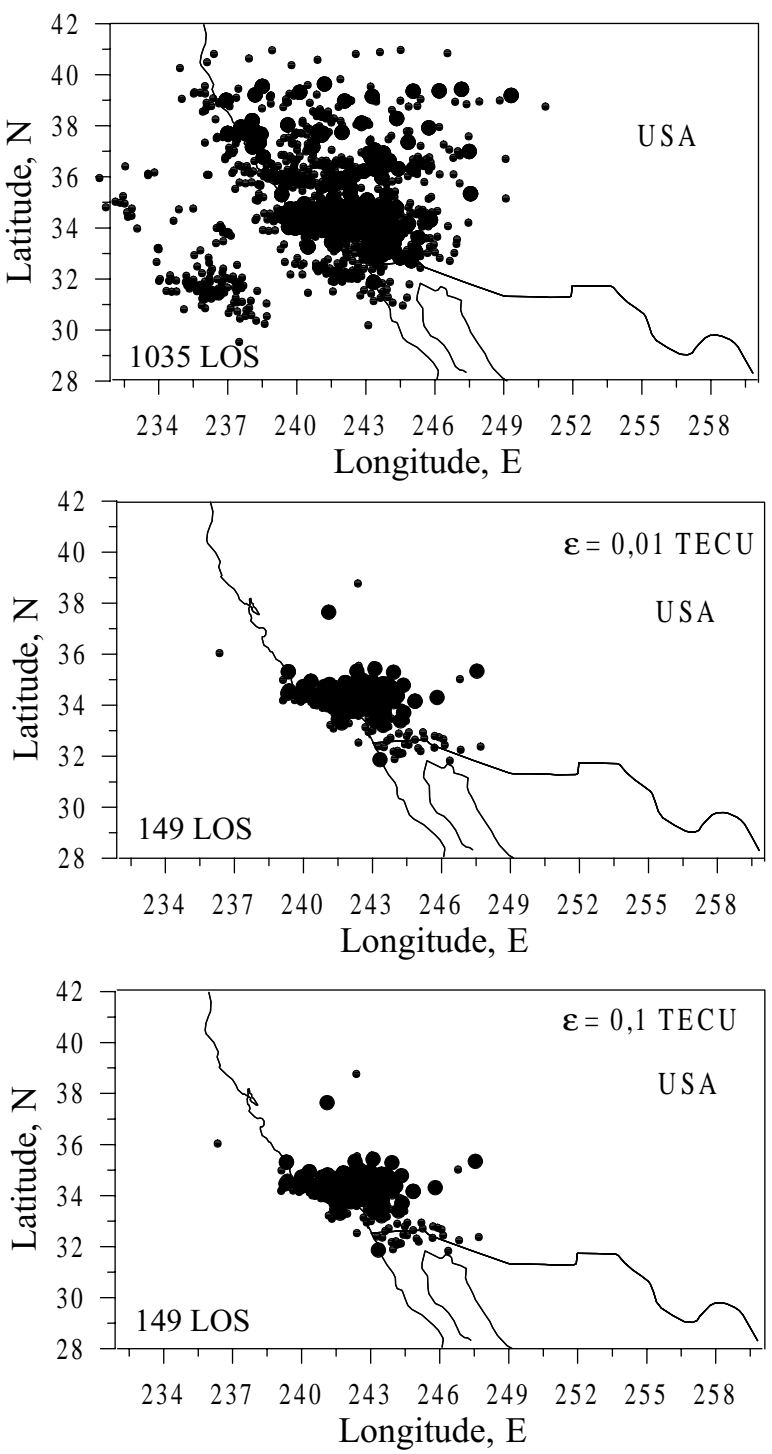

Fig. 4. Geometry of the experiment on detection of TECS on 5 October 2001 in California, USA, from 08:00 to 10:00 UT. Heavy dots show the GPS stations, and small dots show the location of subionospheric points for receiver-satellite Lines-of-Sight. Panel (a) shows the stations and subionospheric points for all Lines-of-Sight. Panels (b) and (c) show the stations and subionospheric points where the TEC variations revealed TECS with an amplitude exceeding the specified threshold $\epsilon=0.01 T E C U(\mathrm{~b})$, and $\epsilon=0.1 T E C U$ (c). Numbers in all panels show the total number of Lines-of-Sight shown in the panel.

The availability of a large number of stations in some regions on the globe, for instance, in California, USA, and West Europe, furnishes an opportunity to determine not only the temporal, but also the spatial characteristics of TECS. In order to estimate the radius of the spatial correlation of events of this type, the number of cases was calculated where TECS within a single $2.3 \mathrm{~h}$ time interval were observed at any two
GPS stations spaced by a distance $d R$ (Fig. 3b). It was found that the localization of TECS in space is sharply defined. In $90 \%$ of the cases the distance $d R$ does not exceed $500 \mathrm{~km}$.

The event of 5 October 2001 was used in the analysis of the TECS dynamic characteristics. On that day, between 08:00 and 18:00 UT, a number of GPS stations located in California, USA $\left(220-260^{\circ} \mathrm{E} ; 28-42^{\circ} \mathrm{N}\right)$ recorded a large number of traveling ionospheric disturbances (TIDs) of the TECS type. For the above-mentioned time interval and the selected longitude range, the local time varied from 00:00 to 10:00 LT (for the longitude of $240^{\circ} \mathrm{E}$ corresponding to the center of the GPS station array used in the analysis). So that the experimental conditions were characteristic for the nighttime ionosphere.

Figure 4 illustrates the geometry of the experiment on 5 b. October 2001. Since several (at least four) GPS satellites are observed at each receiving site simultaneously, the number of Lines-of-Sight far exceeds the number of stations, which enhances considerably the possibilities of analysis. Panel (a) presents the entire set of GPS stations and subionospheric points that were used in the experiment for the time interval from 08:00 to 10:00 UT; panels (b) and (c) - the same, but for the specified threshold $\epsilon=0.01 T E C U$ (b), and $\epsilon=0.1 T E C U$ (c). Except in a single case, TECS were recorded along the LOS running over land. As is evident from the figure, the increase in the recording threshold did not change the number of events recorded.

Figure $5 \mathrm{a}-\mathrm{c}$ presents the geomagnetic variations on $5 \mathrm{Oc}-$ tober 2001. At the lower time scale in Fig. 5, the local time LT is represented for the longitude of $240^{\circ}$ E. Figure $5 \mathrm{~d}$ presents the distribution of the values of the GPS station latitudes and time $t_{\min }$. The letters A, B, C, and D in Fig. $5 \mathrm{~d}$ label the TECS "traces" that are presented in Fig. 6 on a smaller time scale. Figure $5 \mathrm{e}-$ same as in Fig. $5 \mathrm{~d}$, but for the station longitudes and $t_{\min }$. Figure $5 \mathrm{f}$ presents the distribution $N(t)$ of the number of TECS that were detected that day at all GPS stations used in the analysis.

\section{The characteristics of IIDs as deduced from the 5 Oc- tober 2001 event over California, USA}

4.1 Determining the dynamic characteristics of IIDs by the SADM-GPS method

The methods of determining the form and dynamic characteristics of TIDs that are used in this study are based on those reported in Afraimovich (1997) and Afraimovich et al. (1998, 1999, 2000c).

The received phase of the transionospheric radio signal is given by

$\phi=8,42 \times 10^{-7} I / f+\phi_{0}$,

where $\phi$ is the phase (rad) measured at the receiver, $f$ is the received frequency $(\mathrm{Hz}), \phi_{0}-$ the initial phase (Spoelstra and Kelder, 1984). Then the phase differences $\Delta \phi$ along the axes $x$ (directed to the east) and $y$ (directed to the north) 

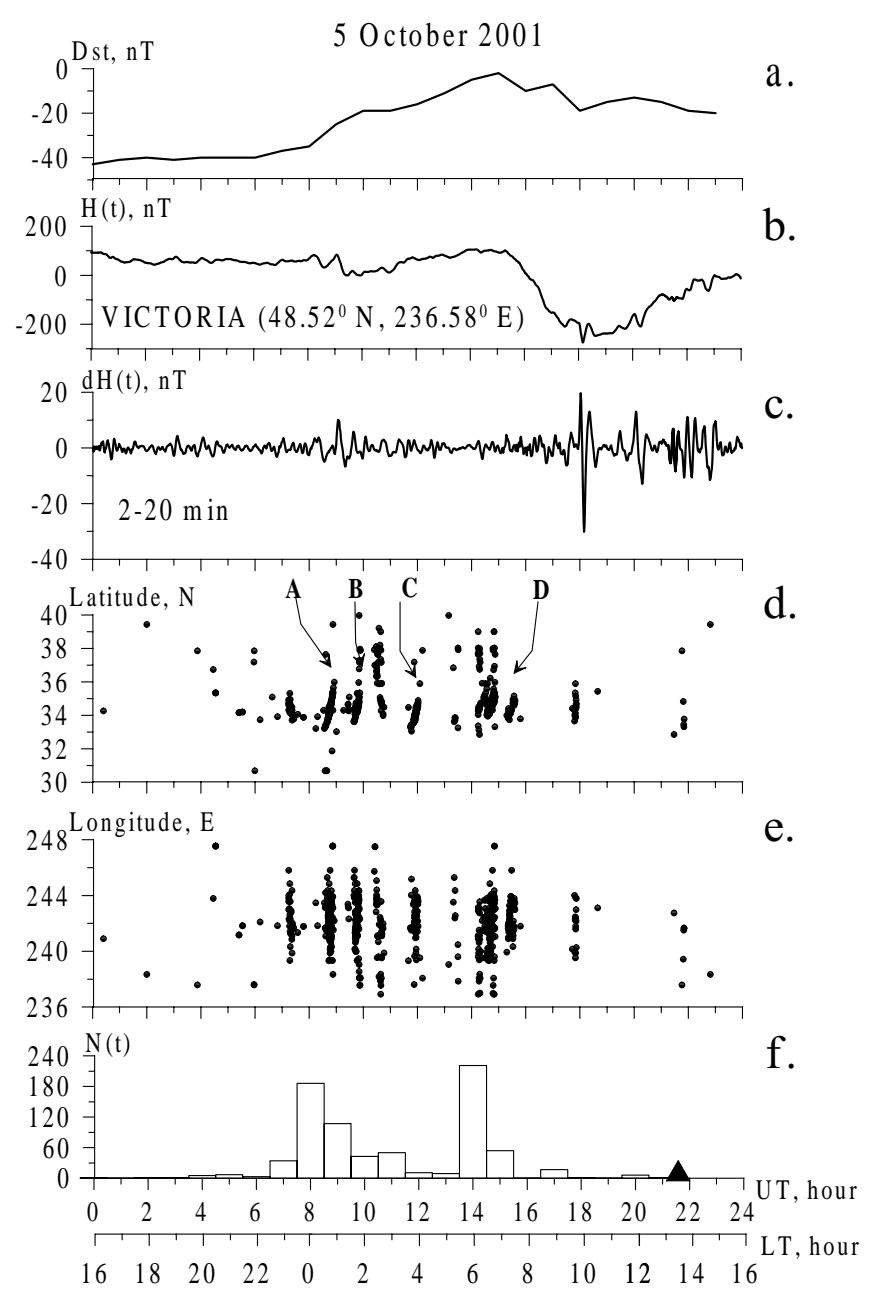

Fig. 5. Geomagnetic field $D_{s t}$-variations (a) 5 October 2001. $H(t)$ variations of the horizontal component of the geomagnetic field as recorded at station Victoria $\left(236.58^{\circ} \mathrm{E} ; 48.52^{\circ} \mathrm{N}\right)-(\mathbf{b}) . d H(t)$ variations, filtered from the series $H(t)$ in the range of periods of 2-20 min - (c). (d) - the distribution of the values of the GPS station latitudes from time $t_{\mathrm{min}}$, corresponding to each of the TECS detected that day by all GPS stations of the California region that were used in the analysis $\left(220-260^{\circ} \mathrm{E} ; 28-42^{\circ} \mathrm{E}\right) ;(\mathrm{e})-$ same as in (d), but for the station longitudes and $t_{\min } ;(\mathbf{f})-$ the distribution $N(t)$ of the number of TECS that were detected that day at all GPS stations used in the analysis, with the rms above $\epsilon=0.1 T E C U$. The letters A, B, C, and D in (d) label the TECS "traces" that are presented in Fig. 6 on a smaller time scale. The triangle in panel (f) designates the Titan-4 rocket launch time 21:21 UT from the spacelaunch complex "Vandenberg" $\left(239.5^{\circ} \mathrm{E} ; 34.8^{\circ} \mathrm{N}\right)$.

are proportional to the values of the horizontal components $G_{E}=I_{x}^{\prime}$ and $G_{N}=I_{y}^{\prime}$ of TEC gradient; primes denote derivatives with respect to a variable specified by the lower index. For convenience of presentation, we will be using values of phase as well as TEC.

The correspondence of space-time phase characteristics, obtained through transionospheric soundings, with local characteristics of disturbances in the ionosphere, was considered in detail in a wide variety of publications (Bertel et al.,
1976; Afraimovich et al., 1992; Mercier and Jacobson, 1997) and is not analyzed at length in this study. The most important conclusion of the cited references is the fact that, as for the extensively exploited model of a "plane phase screen", disturbances $\Delta I(x, y, t)$ of TEC faithfully copy the horizontal part of the corresponding disturbance $\Delta N(x, y, z, t)$ of local concentration, independently of the angular position of the source, and can be used in experiments on measuring the wave disturbances of TEC.

We determine the velocity and direction of motion of the phase interference pattern (phase front) in terms of some models of this pattern, an adequate choice of which is of critical importance. In the simplest form, space-time variations in TEC variations $d I(t, x, y)$ in the ionosphere that are proportional to the phase variation of the transionospheric radio signal, at each given time $t$, can be represented in terms of the phase interference pattern that moves without a change in its shape (the non dispersive disturbances):

$I(t, x, y)=F\left(t-x / u_{x}-y / u_{y}\right)$,

where $u_{x}(t)$ and $u_{y}(t)$ are the displacement velocities of intersection of the phase front of the axes $x$ and $y$, respectively, and $F$ is an arbitrary function.

A special case of Eq. (4) is the most often used model for a solitary plane travelling wave of TEC disturbance

$I(t, x, y)=\delta \sin \left(\Omega t-K_{x} x-K_{y} y+\varphi_{0}\right)$,

where $\delta, K_{x}, K_{y}, \Omega$-respectively, are the amplitude, the $x$ and $y$-projections of the wave vector $\boldsymbol{K}$, and the angular frequency of the disturbance; $\varphi_{0}$ is the initial disturbance phase; $\boldsymbol{K}$ is the horizontal projection of full wave vector $\boldsymbol{K}_{\boldsymbol{t}}$.

Figure $7 \mathrm{a}$ gives a schematic representation of the transionospheric sounding geometry (see caption). It should be noticed, however, that in real situations neither of these ideal models (Eqs. 4 and 5) are realized in a pure form. This is because the TIDs propagate in the atmosphere in the form of a dispersing wave packet with a finite value of the width of the angular spectrum. But in the first approximation on a short time interval of averaging, compared to a time period of filtered variations of TEC, the phase interference pattern moves without a substantial change in its shape.

A Statistical, Angle-of-arrival and Doppler Method (SADM) was proposed by Afraimovich (1997) for determining the characteristics of the dynamics of the phase interference pattern in the horizontal plane by measuring variations of TEC derivatives with respect to the spatial coordinates $I_{x}^{\prime}(t), I_{y}^{\prime}(t)$, and to the time $I_{t}^{\prime}(t)$. This permits the determination of the unambiguous orientation of $\alpha(t)$ of the wave-vector $\boldsymbol{K}$ in the range $0-360^{\circ}$ and the horizontal velocity $u(t)$ at each specific instant of time

$$
\begin{aligned}
\alpha(t) & =\arctan \left(I_{x}^{\prime}(t) / I_{y}^{\prime}(t)\right) \\
u_{x}(t) & =I_{t}^{\prime}(t) / I_{x}^{\prime}(t)=u(t) / \sin \alpha(t) \\
u_{y}(t) & =I_{t}^{\prime}(t) / I_{y}^{\prime}(t)=u(t) / \cos \alpha(t) \\
u(t) & =\left|u_{x}(t) u_{y}(t)\right| /\left(u_{x}^{2}(t)+u_{y}^{2}(t)\right)^{1 / 2} .
\end{aligned}
$$


5 October 2001
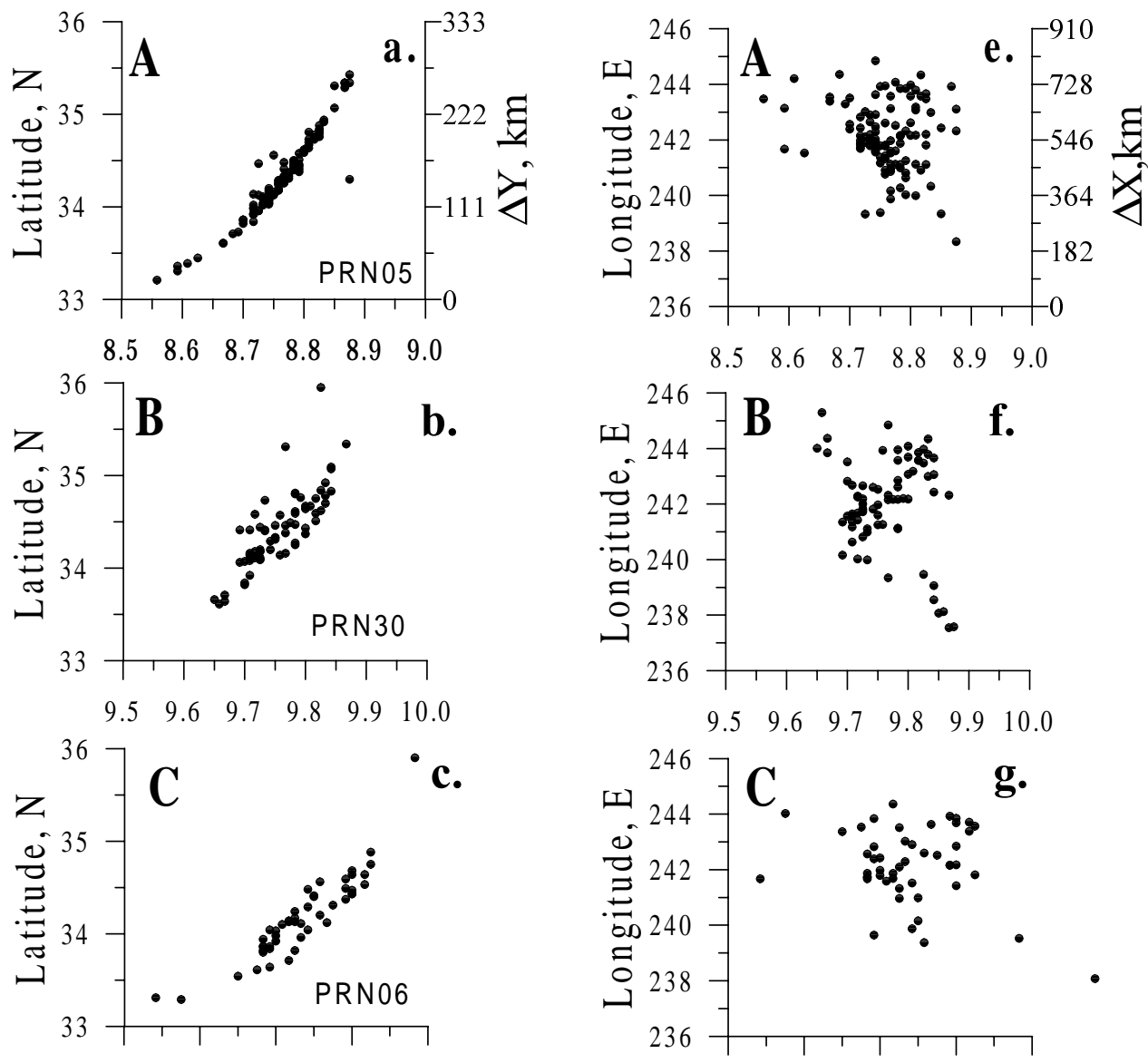

c.
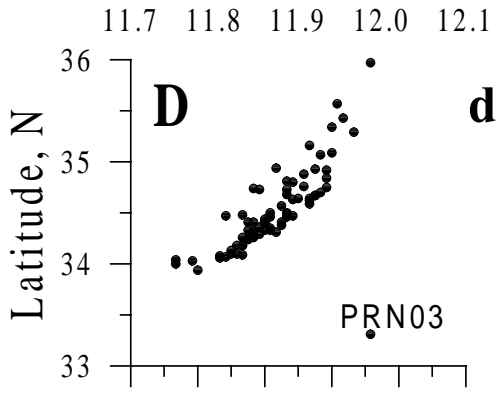

14.514 .614 .714 .814 .915 .0

UT, hour

d.

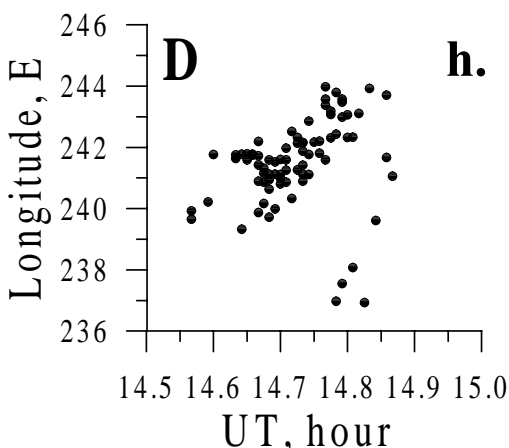

Fig. 6. (a), (c), (e), (g) - The distribution of the values of the GPS station latitudes from time $t_{\min }$, corresponding to each of the TECS detected that day; the letters A, B, C and D label the TECS "traces" that are presented in Fig. 5 on a longer time scale; (b), (d), (f), (h) - same as in Fig. 8a, c, e, and g, but for the station longitudes and $t_{\min }$. The right scales on panels (a) and (e) shows the relative linear coordinates $\Delta X$ and $\Delta Y$ in topocentric frame of reference.

However, direct use of the SADM algorithm that was developed for experiments with geostationary satellites is unacceptable in GPS radio interferometry of TIDs, as this neglects the displacement of LOS on satellites with respect to TIDs in the ionosphere. To allow for this effect in view of the integral character of TEC measurements at a transionospheric sounding, in the first approximation, it is possible to invoke a sim- ple model for an equivalent "thin phase screen" located in a horizontal plane at the height of the main maximum of electron density $h_{\max }$ (Afraimovich et al., 1998, 1999, 2000c). This is quite acceptable because the wavelength of TIDs is comparable to the height scale in the region of the main maximum, which does make the main contribution to the phase modulation caused by the displacement of TIDs. In other 


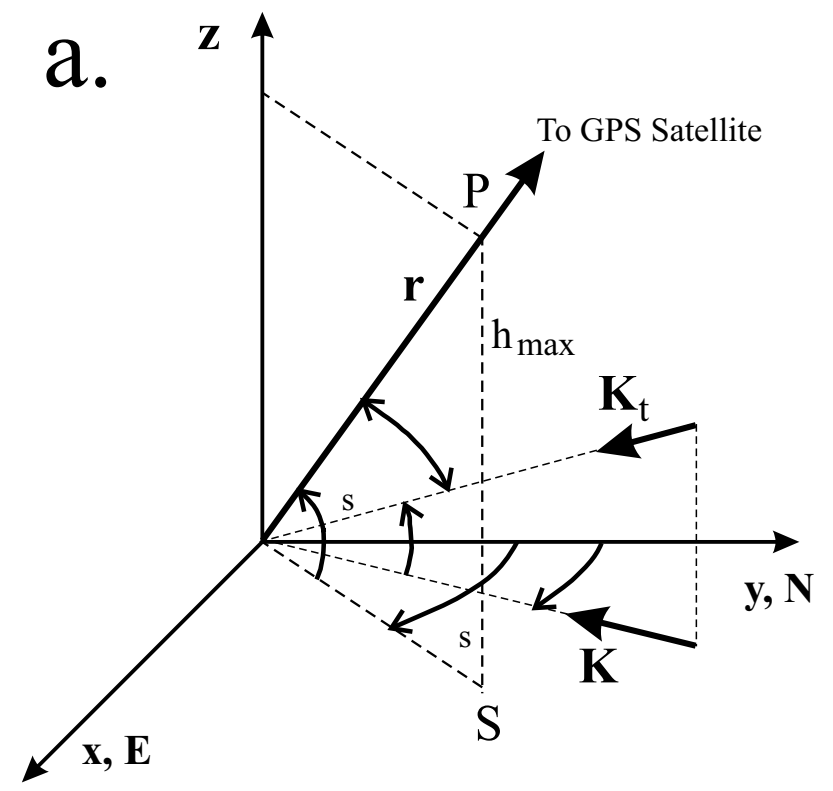

b.

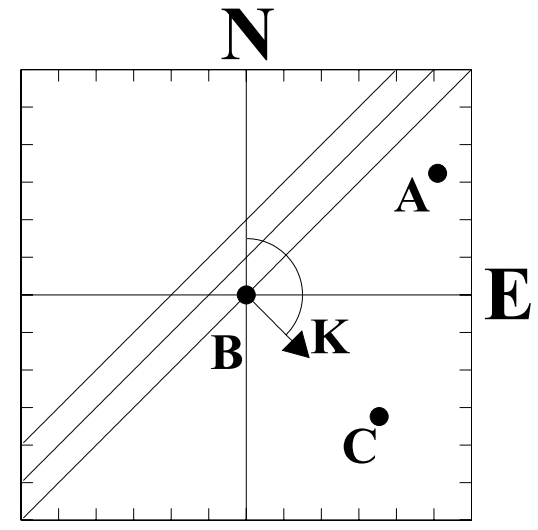

Fig. 7. (a) - schematic representation of the transionospheric sounding geometry. The axes $z, y$, and $x$ are directed, respectively, zenithward, northward (N) and eastward (E). P - point of intersection of LOS to the satellite with the maximum of the ionospheric F2-region; S subionospheric point; $\alpha_{S}$ - the azimuthal angle, counted off from the northward in a clockwise direction; and $\theta_{S}-$ the angle of elevation between the direction $\mathbf{r}$ along LOS and the terrestrial surface at the reception site; $\gamma-$ the angle between the vectors $\boldsymbol{K}_{\boldsymbol{t}}$ and $\boldsymbol{r}$. (b) - GPSarray geometry; A, B, C - receiver points, at which two-frequency multichannel GPS-receivers are installed. Parallel lines correspond to lines of equal TEC. The arrow indicates the vector $\boldsymbol{K}$ in the direction $\alpha$.

words, the imaginary detector is located at the point $P$ of intersection of the LOS to the satellite with an arbitrary plane selected at a height $h_{\max }$ of the electron density peak in the ionosphere. The subionospheric point $S$ is a projection of the point $P$ onto the terrestrial surface (Fig. 7a).

Afraimovich et al. $(1998,1999,2000 \mathrm{c})$ described the updating of the SADM algorithm for GPS-arrays (SADM-GPS) based on a simple model for the displacement of the phase interference pattern that travels without a change in the shape, and on using current information about the angular coordinates of the LOS: the elevation $\theta_{s}(t)$ and the azimuth $\alpha_{S}(t)$. Since the distance between GPS array elements (tens of and a few hundred $\mathrm{km}$ ) is much smaller than the distance to the GPS satellite (over $20000 \mathrm{~km}$ ), the array geometry at a height $h_{\max }$ is similar to the geometry of the array at the ground level. Consequently, one can consider the motion of the imaginary detectors with respect to the ground-based GPS stations in one plane. Clearly, such an approximation of the flat earth corresponds to large values of the elevation of the LOS to the satellite; this condition is satisfied in this paper.

We now introduce a fixed coordinate system $(x O y)$ tied to ground-based stations, centered on the point $O$ (Fig. 8). An infinite plane front $T E C=$ const (Fig. 8, thick line) moves in this coordinate system with the phase velocity $V_{h}$. The coordinate system $\left(x^{\prime} S y^{\prime}\right)$, tied to the subionospheric point $S$, moves with respect to the system $(x O y)$, with the velocity $w$ (Fig. 8). The coordinates $x_{s}(t)$ and $y_{s}(t)$ of the intersection point $P$ at $h_{\max }$ in the chosen topocentric coordinate system
(Fig. 7a) vary as

$x_{s}(t)=h_{\max } \sin \left(\alpha_{s}(t)\right) \cot \left(\theta_{s}(t)\right)$

$y_{s}(t)=h_{\max } \cos \left(\alpha_{s}(t)\right) \cot \left(\theta_{s}(t)\right)$

and the $x$ - and $y$-components of the displacement velocity $w$

$w_{x}(t)=\left(x_{s}(t)\right)_{t}^{\prime}$

$w_{y}(t)=\left(y_{s}(t)\right)_{t}^{\prime}$.

The equations for the transition from the fixed coordinate system $(x O y)$ to a moving coordinate system $\left(x^{\prime} S y^{\prime}\right)$ via a parallel transfer of the coordinate axes are written as (Korn and Korn, 1968):

$x^{\prime}=x-w_{x} t$

$y^{\prime}=y-w_{y} t$

Since the transformation of coordinates is accomplished without rotation, the direction of travel of the front line in a fixed $(x O y)$ and moving $\left(x^{\prime} S y^{\prime}\right)$ coordinate systems remains unchanged. The equation for the front line $T E C=$ const in a moving coordinate system $\left(x^{\prime} S y^{\prime}\right)$ may be represented as a normal equation for a straight line (Korn and Korn, 1968)

$x^{\prime} \sin \alpha+y^{\prime} \cos \alpha-u t=0$,

where $u$ is the velocity of travel of the front line in a direction normal to the front line ( $T E C=$ const $)$, i.e. in the direction of the wave vector $\boldsymbol{K}$ in a moving coordinate system $\left(x^{\prime} S y^{\prime}\right)$, and $u t$ is the distance from the point $S$ to the front line.

Substituting Eq. (9) into Eq. (10) gives the equation for front line in a fixed coordinate system $(x O y)$ :

$x \sin \alpha+y \cos \alpha-\left(u+w_{x} \sin \alpha+w_{y} \cos \alpha\right) t=0$, 


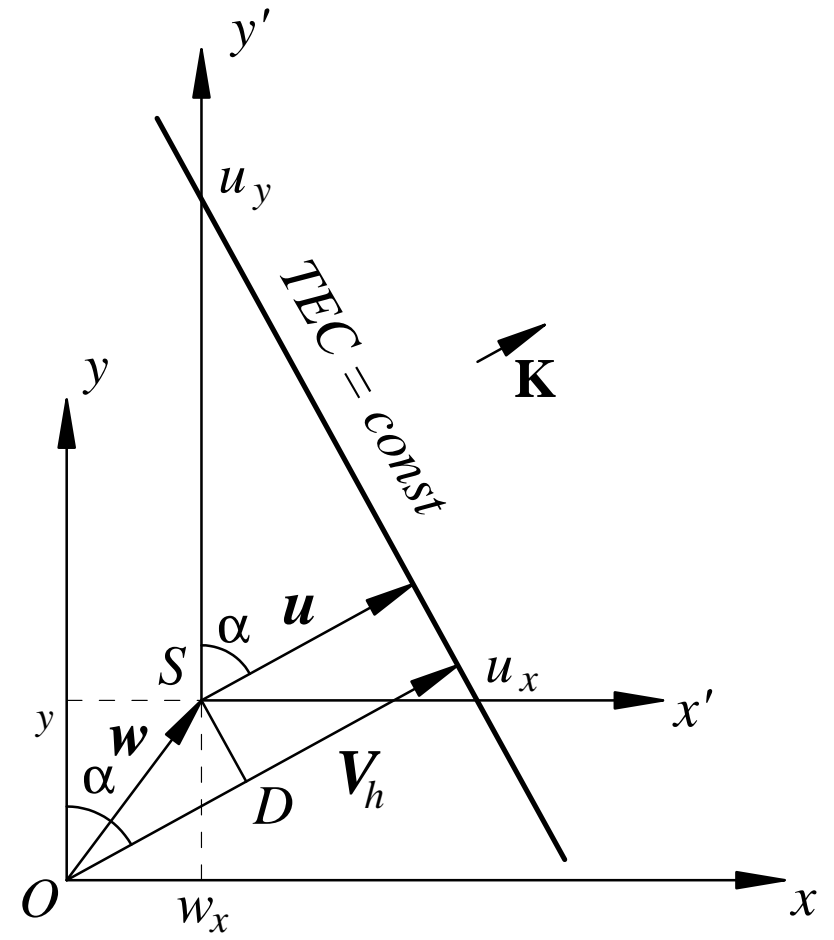

Fig. 8. The moving radio interferometer coordinate system $\left(x^{\prime} S y^{\prime}\right)$ and the fixed coordinate system $(x O y): u_{x}^{\prime}$ and $u_{y}^{\prime}$ are the velocities of intersection of the line $T E C=$ const of the axes $x^{\prime}$ and $y^{\prime}$ in a moving coordinate system $\left(x^{\prime} S y^{\prime}\right)$ and $w_{x}, w_{y}$ are the $x$ - and $y$ projections of the satellite displacement velocity $w$.

from when we have the ultimate expression for the absolute value of the velocity of the front line $V_{h}$ in a fixed coordinate system $(x O y)$ :

$V_{h}=u+w_{x} \sin \alpha+w_{y} \cos \alpha$.

Equation (12) can be obtained in a different way by taking into consideration that the modulus of the phase velocity $V_{h}$ equals the sum of the velocity modulus $u$ and the projection $O D$ of the vector $\boldsymbol{w}$ onto the direction of the wave vector $\boldsymbol{K}$ (see Fig. 8)

$O D=w_{x} \sin \alpha+w_{y} \cos \alpha$.

Let us consider some special cases related to the application of Eq. (12). Assume that the velocity vector $\boldsymbol{w}=$ $\left(w_{x}, w_{y}\right)$ of the subionospheric point is perpendicular to the wave vector $\boldsymbol{K}$. In other words, it is assumed that the subionospheric point drifts along the constant-phase lines; then the value of the velocity $\boldsymbol{w}$ must not influence the relationship between $u$ and $V_{h}$.

Let us check to see that this is the case in Eq. (12). As an example, we specify $\alpha=0$. Then $\mathbf{K}=(0, K)$ and $\boldsymbol{w}=(w, 0)$. Substituting into Eq. (12) gives $V_{h}=u+$ $w \sin 0+0 \cos 0=u$. This means that for the case under consideration, the value of the velocity $w$ does not have any influence on the value of the velocity $V_{h}$ in a fixed coordinate system.
In the case where the directions of the vectors $\boldsymbol{K}$ and $\boldsymbol{w}$ coincide, the resulting value of $V_{h}$ equals the sum

$V_{h}=u+w$.

In this case, if the subionospheric point catches up with the front $T E C=$ const, the measured value of the velocity $u$ becomes less than the true $V_{h}$. If the subionospheric point moves in the direction opposite to the front $T E C=$ const, the measured value of $u$ is larger than the true value of $V_{h}$.

Let us take a brief look at the sequence of data handling procedures. Out of a large number of GPS stations, three points $(A, B, C)$ are chosen in such a way that the distances between them do not exceed about one-half the expected wavelength $\Lambda$ of the disturbance (see Fig. 7b). The point $B$ is taken to be the center of a topocentric frame of reference. Such a configuration of the GPS receivers represents a GPS-array (or a GPS-interferometer) with a minimum of the necessary number of elements. In regions with a dense network of GPS-points, we can obtain a broad range of GPSarrays of a different configuration, which furnishes a means of testing the data obtained for reliability; in this paper we have taken advantage of this possibility (Sect. 4.2).

The input data include a series of the vertical TEC $I_{A}(t)$, $I_{B}(t), I_{C}(t)$. Linear transformations of the differences of the values of the filtered TEC variations $\left(d I_{\mathrm{B}}-d I_{\mathrm{A}}\right)$ and $\left(d I_{\mathrm{B}}-\right.$ $\left.d I_{\mathrm{C}}\right)$ at the receiving points $\mathrm{A}, \mathrm{B}$ and $\mathrm{C}$ are used to calculate the components of the TEC gradient $I_{x}^{\prime}$ and $I_{y}^{\prime}$ (Afraimovich et al., 1998). The time derivative of TEC $I_{t}^{\prime}$ is determined by differentiating $I_{\mathrm{B}}(t)$ at the point $\mathrm{B}$.

The resulting series are used to calculate instantaneous values of the horizontal velocity $V_{h}(t)$ and the azimuth $\alpha(t)$ of TID propagation. Next, the series $V_{h}(t)$ and $\alpha(t)$ are put to a statistical treatment. This involves constructing distributions of the horizontal velocity $P\left(V_{h}\right)$ and direction $P(\alpha)$ which are analyzed to test the hypothesis of the existence of the preferred propagation direction. If such a direction does exist, then the corresponding distributions are used to calculate the mean value of the horizontal velocity $\left\langle V_{h}\right\rangle$, as well as the mean value of the azimuth $\langle\alpha\rangle$ of TID propagation.

The TEC response amplitude experiences a strong "aspect" dependence caused by the integral character of a transionospheric sounding (Afraimovich et al., 1992). As a first approximation, the transionospheric sounding method is responsive only to TIDs with the wave vector $\boldsymbol{K}_{\boldsymbol{t}}$ perpendicular to the direction $\boldsymbol{r}\left(\gamma=90^{\circ}\right.$; see Fig. 7a). A corresponding condition for elevation $\theta$ and azimuth $\alpha$ of an arbitrary wave vector $\boldsymbol{K}_{\boldsymbol{t}}$ normal to the direction $\boldsymbol{r}$ has the form

$\theta=\arctan \left(-\cos \left(\alpha_{s}-\alpha\right) / \tan \theta_{s}\right)$

We used Eq. (15) in determining the elevation $\theta$ of $\boldsymbol{K}_{\boldsymbol{t}}$ from the known mean value of azimuth $\alpha$ by Afraimovich et al. (1998).

The phase velocity modulus $V$ can be defined as

$V=V_{h} \times \cos (\theta)$. 
Table 1. Mean IID parameters on 5 October 2001.

\begin{tabular}{cccccccc}
\hline & & $\langle A\rangle$, & $\left\langle\left\langle V_{h}\right\rangle\right\rangle$, & & & $\langle\langle V\rangle\rangle$, \\
Trace & $\mathrm{M}$ & $T E C U$ & $\mathrm{~m} / \mathrm{s}$ & $\langle\langle\alpha\rangle\rangle,{ }^{\circ}$ & $\langle\langle\theta\rangle\rangle,{ }^{\circ}$ & $\mathrm{m} / \mathrm{s}$ & $C$ \\
\hline A & 660 & 0.26 & 171 & 0 & 22 & 153 & 8.2 \\
B & 280 & 0.28 & 184 & 5 & 27 & 160 & 7.6 \\
C & 376 & 0.36 & 158 & 5 & 24 & 139 & 10.2 \\
D & 280 & 0.34 & 73 & 0 & 20 & 59 & 6.7 \\
\hline
\end{tabular}

Hence, at least the dynamic (two-dimensional) characteristics of TEC disturbances (TECS in the case under consideration) may well be referred qualitatively to the corresponding characteristics $d N / N$ of local electron density disturbances (IIDs). Reconstructing the quantitative characteristics of local density disturbances, $d N / N$, in terms of the solution of an inverse problem of transionospheric sounding is a highly difficult, special problem, constituting the subject of our further investigation.

\subsection{Results of experiment}

On the basis of using the transformations described in Sect. 4.1, for each of the GPS arrays chosen for the analysis we obtained maximum values of the TECS amplitude $\left|A_{\min }\right|$ and the average for the selected time interval values of the following IID parameters: $\langle\alpha\rangle$ and $\langle\theta\rangle-$ the azimuth and elevation of the wave vector $\boldsymbol{K}_{t} ;\left\langle V_{h}\right\rangle$ and $\langle V\rangle$ - the horizontal component and the phase velocity modulus.

For the PRN05 and PRN30 satellites, Figs. 1c and $\mathrm{f}$ give an example of the filtered TECS for different spaced GPS stations. It is evident from the figure that the selected typical TEC variations such as TECS are identical and shifted by a certain amount of delay, which makes it possible to calculate the velocity and direction of the IID that causes the observed TEC variations. The panels show the values of the IID velocity $V_{h}$ and direction $\alpha$ inferred using the above data processing procedures.

Next, the series of $\left|A_{\min }\right| ;\langle\alpha\rangle ;\langle\theta\rangle ;\left\langle V_{h}\right\rangle$ and $\langle V\rangle$ for different sets of GPS arrays for the entire California region are put to a statistical treatment. This involves constructing distributions of the horizontal component $P\left(V_{h}\right)$ and the phase velocity modulus $P(V)$; the azimuth $P(\alpha)$ and elevation $P(\theta)$ of the wave vector $\boldsymbol{K}_{\boldsymbol{t}}$, presented in Figs. 9 and 10.

Using different sets of GPS arrays we were able to obtain the average for the selected sets of GPS array values of amplitude $\langle A\rangle$; velocity modulus $\langle\langle V\rangle\rangle$; horizontal projection $\left\langle\left\langle V_{h}\right\rangle\right\rangle$ and direction $\langle\langle\alpha\rangle\rangle$ in the horizontal plane, as well as the elevation angle $\langle\langle\theta\rangle\rangle$ of the displacement vector $\boldsymbol{K}_{\boldsymbol{t}}$ in the vertical plane. Corresponding values of the IID parameters, and also numbers of the GPS array $M$ for "traces" A, B, C, $\mathrm{D}$ are presented in Table 1.

An analysis of the distribution of the azimuths $P(\alpha)$ (Figs. 9b and e, 10b and e) shows a clearly pronounced north- ward direction of TECS displacement. The elevation of the TECS wave vector, determined from the aspect condition (15), has mostly a small positive value (Figs. 9c and f, 10c and f). The horizontal component and the modulus of the phase velocity were found to be $V_{h}=70-180 \mathrm{~m} / \mathrm{s}$ and $V=60-160 \mathrm{~m} / \mathrm{s}$.

Let us consider in greater detail the "traces" A, B, C, and D. Figures $6 \mathrm{a}-\mathrm{d}$ suggest the conclusion that at each given time $t_{\min , i}$ there are only a few TECS with close values of the coordinates $N_{i}$, and there is a clearly pronounced gradual displacement of the subionospheric point in the northward meridional direction. On the other hand, the dependencies of the longitude $E_{i}$ of the TECS observed on $t_{\min , i}$ suggest a spatial extent of the traveling irregularity in longitude (Figs. 6e-h). Thus, the shape of the projection of the observed irregularities ("traces" A, B, C, and D) onto the horizontal plane show up as an ellipse.

Coordinates $N_{i}$ and $E_{i}$ can readily be recalculated to the values of the relative linear coordinates $\Delta X_{i}$ and $\Delta Y_{i}$ in a topocentric frame of reference (see right scales on Figs. $6 \mathrm{a}$ and e). The values of $\Delta X_{i}$ have been calculated for the mean value of latitude $N=34.5^{\circ}$. Since the propagation direction $\alpha$ is close to $0^{\circ}$, the ratio $C$ of the axes of the ellipse can be estimated from the ratio $C=\sigma_{X} / \sigma_{Y}$, where $\sigma_{X}$ and $\sigma_{Y}$ are the standard deviations of the corresponding series of the TECS coordinates $X_{i}$ and $Y_{i}$, presented in Fig. 6. Corresponding values of the ratio $C$ are presented in Table 1 . The mean (for "traces" A, B, C and D) ratio $C$ of the axes of the ellipse is 8 .

With a knowledge of the range of TECS displacement in latitude $\left(Y_{i}\right)$ and the time interval corresponding to this displacement, it is easy to determine that the meridional projection of the displacement velocity $V_{N}$ is close to $200 \mathrm{~m} / \mathrm{s}$, which is in agreement with estimates obtained using the SADM-GPS method.

We now estimate the horizontal sizes of the IID. With the mean duration of $10 \mathrm{~min}$ and the travel velocity $V_{h}$ of $160 \mathrm{~m} / \mathrm{s}$, we obtain the transverse size of the irregularity equal to $100 \mathrm{~km}$; the longitudinal size equal to $800 \mathrm{~km}$.

Let us now obtain the mean estimate of the relative amplitude of a local electron density disturbance typified by the IID of 5 October 2001.

A mean TECS absolute amplitude over California equals 0.3 TECU (see Table 1). As the background value of $I_{0}$, we 


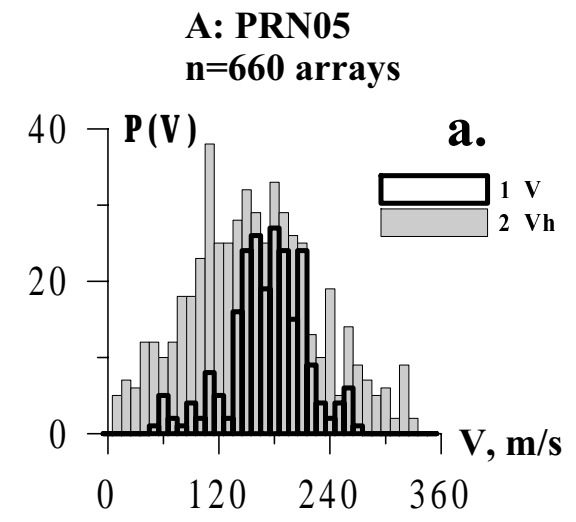

\section{B: PRN30 \\ $\mathrm{n}=\mathbf{2 8 0}$ arrays}
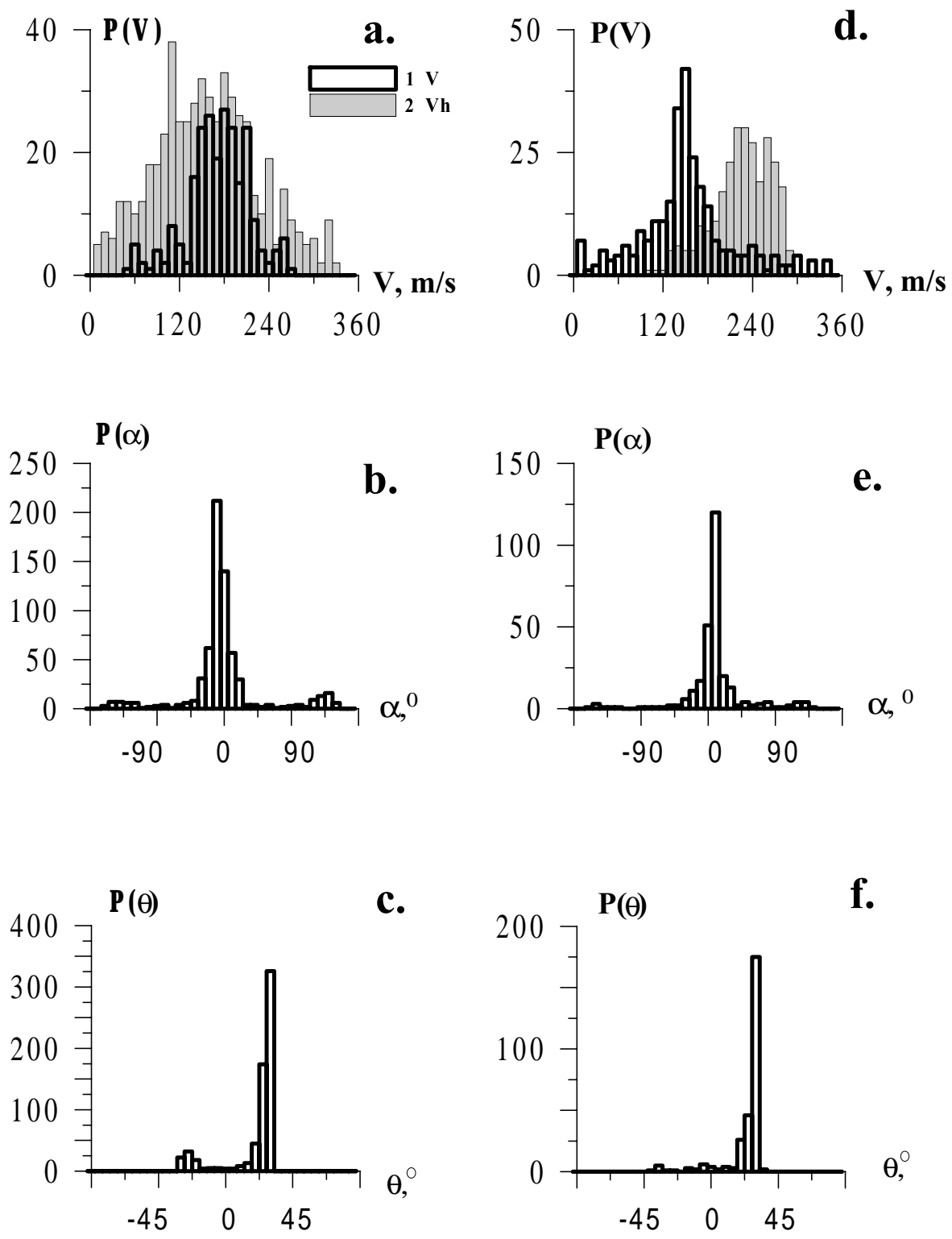

Fig. 9. Distributions of the TECS parameters as determined by the SADM-GPS method, for the "trace" A (on the left; 660 arrays), and for the "trace" B (on the right; 280 arrays). (a), (d) - modulus $V$ (line 1) and horizontal component $V_{h}$ (line 2) of the TECS phase velocity; (b), (e) - azimuth $\alpha$; (c), (f) - elevation $\theta$ of the TECS wave vector $\boldsymbol{K}_{\boldsymbol{t}}$.

used the absolute "vertical" TEC value of $I_{0}(t)$ (see Fig. 11c) obtained from IONEX-maps of the TEC (Mannucci, 1998). These maps with two-hour temporal resolution were constructed using well-known methods and are placed on the Internet site ftp://cddisa.gsfc.naa.gov/.

At the lower time scale in Fig. 11, the local time LT is represented for the longitude of $240^{\circ}$ E. Comparison of Fig. 11c and $d$ reveals that the greatest TECS occurrence probability corresponds to the nighttime hours for which the "vertical" TEC value $I_{0}(t)$ does not exceed $10 T E C U$.

Hence, the relative amplitude of TECS $d I / I_{0}$ makes up 3\%, that is quite significant for this disturbance period $(\Delta T=10 \mathrm{~min})$ and exceeds the amplitude of typical background TEC fluctuations by one order of magnitude(Afraimovich et al., 2001a).

It is further assumed that the characteristic vertical size of the IID is of the same order as the transverse horizontal size (of about $100 \mathrm{~km}$ ). The vertical extent of the part of the ionosphere that makes the main contribution to the TEC modulation is no less than $500-1000 \mathrm{~km}$. Hence, it follows that the relative amplitude $d N / N$ of the local electron density disturbance for IID reaches a considerably large value, $10-20 \%$. 

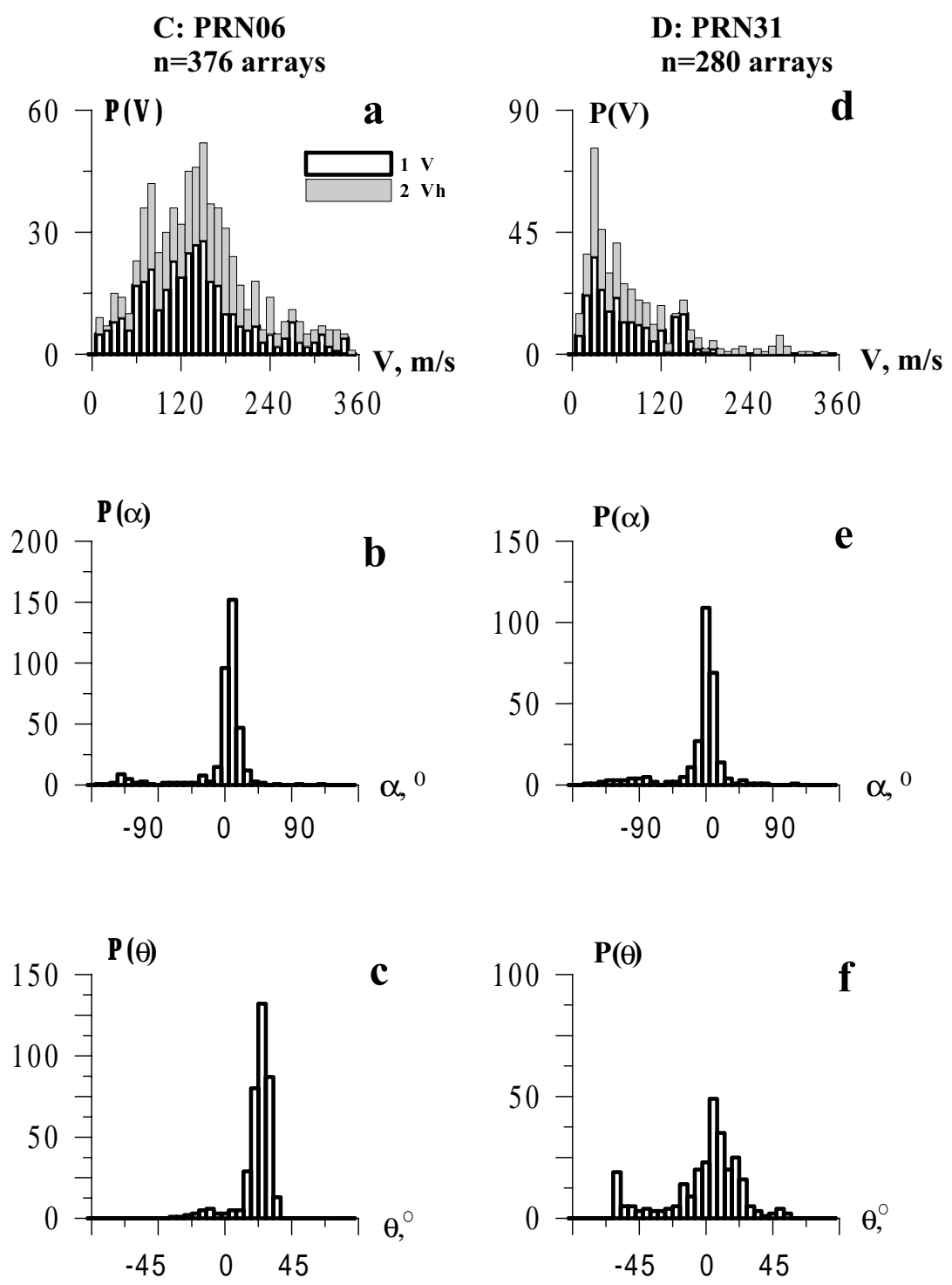

Fig. 10. Same as in Fig. 9, but for the "trace" C (on the left; 376 arrays), and for the "trace" D (on the right; 280 arrays).

\section{Discussion}

What is the nature of the ionospheric irregularities that are responsible for the occurrence of TECS, and do they differ from the known published ionospheric disturbances? We shall try to unravel this situation using the sample statistic obtained in Sect. 3 and the 5 October 2001 event as an example, because for this event it was possible not only to record a large number of TECS, but also to obtain estimates of the size, anisotropy and velocity of isolated ionospheric irregularities that are responsible for the occurrence of TECS (Sect. 4).

It is significant that, according to Internet data for the concerned region of the USA and for the time interval of interest of 5 October 2001, there were not observable meteorological phenomena (site http://www.solar.ifa.hawaii.edu/ Tropical/), powerful explosions or rocket launchings able to cause TEC variations of the TECS type. The Titan-4 rocket was launched from the "Vandenberg" spaceport $\left(239.5^{\circ} \mathrm{E}\right.$; $34.8^{\circ} \mathrm{N}$ ) on 5 October at 21:21 UT (the launching time is shown by the filled triangle in Fig. 5f). However, most TECS on that day were observed before the launching time.

As far as seismic activity is concerned, we analyzed the data covering not only the time interval of our interest, but also for the period from 4 to 20 October 2001 (http: //wwwneic.cr.usgs.gov/neis/FM/previous/0107.html). No earthquakes with a magnitude larger than 3.5 were recorded in the region of our interest for the above time interval.

For 5 October 2001, the time interval of our interest, TEC fluctuations similar to TECS detected over California were absent elsewhere on the globe. No solar flare-induced background fluctuations of the TEC disturbance were also revealed. A similar, quiet, behavior over this time interval was also characteristic for the energetic particle flux. We do not 
present here the relevant data for reasons of space. They may be found on the site http://www.sel.noaa.gov/ftpmenu/lists. html.

The geomagnetic situation on 5 October 2001 can be characterized as a weakly disturbed one, which must lead to some increase in the level of TEC background fluctuations; however, this cannot cause any large-scale changes in electron density which are characteristic for the geomagnetically disturbed ionosphere. In the analysis of the geomagnetic situation we used the data from the magnetic observatory Victoria, which, for the time interval 15:00-18:00 UT of our interest, recorded a geomagnetic disturbance, implying a decrease in the horizontal component of the magnetic field by $100 \mathrm{nT}$ (Fig. 5b).

This disturbance was also accompanied by an increase in the $H$-component fluctuation amplitude in the range of 220-min periods (Fig. 5c). The variation range of the geomagnetic $D_{s t}$-index for the selected time interval was also relatively small (no more than $20 \mathrm{nT}$ ); however, the period from 08:00 to 15:00 UT showed a clearly pronounced increase in variations of the $D_{s t}$-index that coincided with the period when the $H$-component of the magnetic field was increasing (Fig. 5a).

Hence, the data from magnetic-variation stations do not suggest the conclusion that the observed TECSs are associated with magnetic field variations.

5.1 The difference of TECS from the TEC response to shock-acoustic waves generated due to rocket launchings and earthquakes

First of all, it is important to remark that TECS variations are close in amplitude, form and duration to the TEC response to shock-acoustic waves (SAW) generated during rocket launchings (Calais and Minster, 1996; Calais et al., 1998b; Li et al., 1994; Afraimovich et al., 2000a, 2001b), earthquakes (Calais and Minster, 1995; Afraimovich et al., 2001c), and explosions (Fitzgerald, 1997; Calais et al., 1998a). In this connection, TECS can mask TEC responses to technogenic effects and lead to spurious signals recorded in detection systems for such effects, based on analyzing signals from the global GPS network.

Afraimovich et al. (2000a; 2001b, c) found that in spite of a difference in rockets and earthquakes characteristics, the ionospheric TEC response for all such events had the character of an $N$-wave corresponding to the form of a shock wave. The SAW period $\Delta T$ is $270-360 \mathrm{~s}$, and the amplitude exceeds the standard deviation of total electron content background fluctuations in this range of periods under quiet and moderate geomagnetic conditions by factors of 2 to 5 as a minimum. The angle of elevation $\theta$ of the SAW wave vector varies from $30^{\circ}$ to $60^{\circ}$, and the SAW phase velocity $V$ $(900-1200 \mathrm{~m} / \mathrm{s})$ approaches the sound velocity at heights of the ionospheric F-region maximum.

A spatial and temporal processing of data from GPS arrays can be used in selecting these phenomena, in order to estimate the propagation velocity of TEC disturbances. In
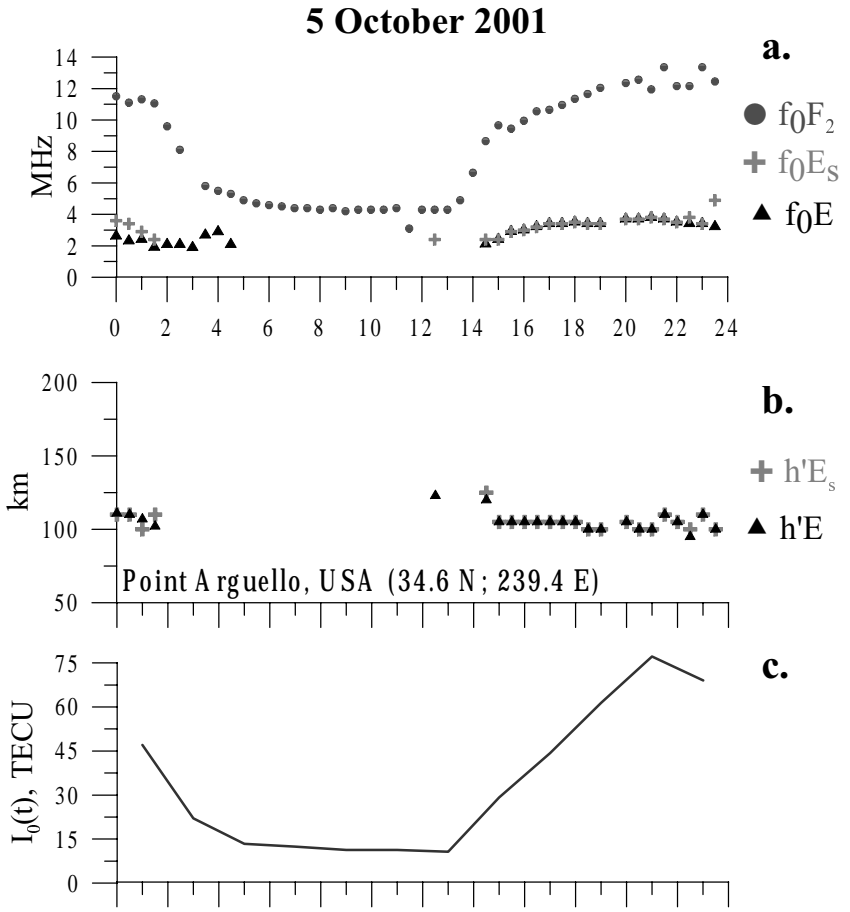

c.

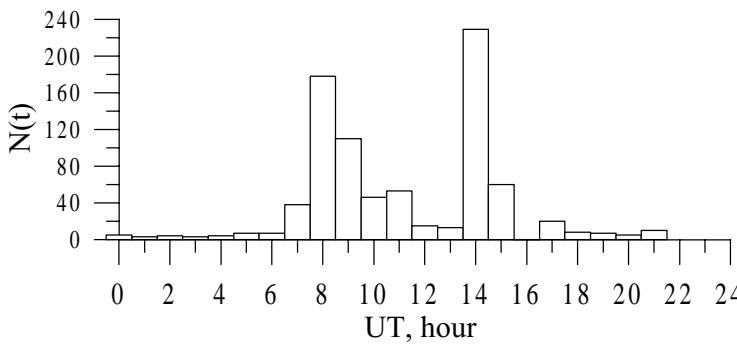

d.

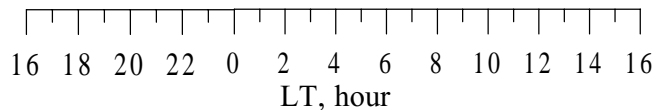

Fig. 11. (a) - Values of the critical frequencies $f o \mathrm{~F} 2$ - heavy dots; fo $E_{S}$ - crosses; foE -shaded triangles, measured on 5 October 2001 at ionospheric station Point Arguello $\left(239.4^{\circ} \mathrm{E} ; 34.6^{\circ} \mathrm{N}\right)$; (b) - variations of virtual heights $h^{\prime} E$ - shaded triangles, $h^{\prime} E_{S}$ - crosses. (c) - variations of the absolute "vertical" TEC value $I_{0}(t)$ for the site with the coordinates $240^{\circ} \mathrm{E} ; 35^{\circ} \mathrm{N}$, obtained from IONEX-maps of TEC; (d) - the same as Fig. $5 f$.

particular, the velocity of IIDs $(150-200 \mathrm{~m} / \mathrm{s})$ is far less than the SAW velocity $(900-1200 \mathrm{~m} / \mathrm{s})$ and is most likely to correspond to the propagation velocity of medium-scale background AGW (Kalikhman, 1980; Afraimovich et al., 1998, 1999; Mercier and Jacobson, 1997; Hocke and Schlegel, 1996; Oliver et al., 1997). The SAW and IID responses can be distinguished using this attribute, as well as from the difference of angular characteristics of the disturbance wave vector (see Sect. 4). 
5.2 Can TECS be caused by the $E_{S}$-layer ionospheric irregularities that are responsible for S- and QPscintillations?

As has been pointed out in the Introduction, a large number of publications (e.g. Karasawa, 1985; Titheridge, 1971; etc.), were devoted to the study the "spikes-type" (S-) scintillations.

Bowman (1989) observed quasi-periodic (QP) oscillations of the signal from an orbiting satellite at $150 \mathrm{MHz}$ frequency. The anticipated cause of the occurrence of such QP oscillations is Fresnel diffraction from ionospheric irregularities. Evidently, irregularities giving rise to QP oscillations reside in the $E_{s}$ level containing a host of small regions (less than $200 \mathrm{~m}$ in size), with high electron density separated from one another by a distance of several tens of kilometers. The occurrence of QP oscillations is peaked in the nighttime hours. The calculated velocities of irregularities and $E_{s}$ structures range from 50 to $100 \mathrm{~m} / \mathrm{s}$.

The cited authors point out that transionospheric signal variations of this kind are observed mostly at night and are most likely caused by the irregularities located in the $E_{s^{-}}$layer. This brings up the question of whether the TECS recorded by us are able to be caused by such ionospheric irregularities. At this point it should be noted that TECS are also observed mostly at night, and their velocities (on the order of $100 \mathrm{~km} / \mathrm{s}$ ) are close to those obtained by Karasawa (1985), Titheridge (1971), and Bowman (1989).

In order to verify the validity of this TECS model for the 5 October 2001 event we availed ourselves of the data (placed on the site http://spidr.ngdc.noaa.gov/spidr/) from the ionospheric station Point Arguello located in the center of the region of our interest.

Figure 11a presents the values of the critical frequencies $f o \mathrm{~F} 2, f o E_{s}$ and $f o \mathrm{E}$, measured on 5 October 2001 at ionospheric station Point Arguello; Figure $11 \mathrm{~b}$ - variations of virtual heights $h^{\prime} E$ and $h^{\prime} E_{s}$.

An analysis of the dependence of critical frequencies and effective heights of reflections from the $F$ - and $E_{s}$-regions showed that during 08:00-14:00 UT, when the largest number of TECS was recorded, reflections from the $E_{s}$-layer were absent altogether (cf. panels (a), (b), (d). Thus, we did not obtain any direct confirmation of the fact that TECS were caused by intense ionospheric irregularities located in the $E_{s}$-layer. The question of the origin of the IID that are responsible for TECS remains open.

It should be noted that direct comparison of our results with the data obtained by Karasawa (1985), Titheridge (1971), and Bowman (1989), is made difficult by the fact that the overwhelming amount of earlier data was obtained for amplitude, rather than phase, scintillations. On the other hand, extracting data on amplitude variations of GPS signals from Internet RINEX-files is highly difficult (Gurtner, 1993).

To understand the nature of TECS requires invoking data from different independent diagnostic tools, including incoherent scatter radars, ionosondes, magnetometers, etc.

\section{Conclusion}

Main results of this study may be summarized as follows:

1. TECS constitute a rare event that occurs mainly in the nighttime in the spring and autumn periods.

2. In the time region, TECS represent single negative aperiodic abrupt changes of TEC with a duration of about 10-20 min; the mean value of the TECS amplitude exceeds the mean value of the "background" TEC variation amplitude by a factor of 5-10 as a minimum.

3. TECS represent a local phenomenon with a typical radius of spatial correlation not larger than $500 \mathrm{~km}$.

4. The TECS we recorded on 5 October 2001, were caused by isolated ionospheric irregularities; the horizontal sizes of the observed irregularities are $100 \mathrm{~km}$ by $800 \mathrm{~km}$. IIDs travel in a direction perpendicular to their elongation (northward in the case under consideration), with the mean velocity $160 \mathrm{~m} / \mathrm{s}$, which corresponds to the velocity of medium-scale AGW (of about $100-200 \mathrm{~m} / \mathrm{s}$ ).

We are aware that this study has revealed only the key averaged patterns of this phenomena, and we hope that it would give impetus to a wide variety of more detailed investigations.

Acknowledgements. We are indebted to N. T. Afanasyev for participating in discussions. We thank O. S. Lesyuta for his help in organizing the experiment. We are also grateful to V. G. Mikhalkovsky for his assistance in preparing the English version of the manuscript. Finally, the authors wish to thank the referees for valuable suggestions which greatly improved the presentation of this paper. This work was done under state support grant No. Nsh-272.2003.5 for Leading Scientific Schools of the Russian Federation as well as Russian Foundation for Basic Research grants No. 00-05-72026 and 03-05-64100. We acknowledge the Scripps Orbit and Permanent Array Center (SOPAC) for providing GPS data used in this study.

Topical Editor M. Lester thanks E. Calais and another referee for their help in evaluating this paper.

\section{References}

Afraimovich, E. L., Terechov, A. I., Udodov, M. Yu., and Fridman, S. V.: Refraction distortions of transionospheric radio signals caused by changes in a regular ionosphere and by travelling ionospheric disturbances, J. Atmos. and Terr. Phys., 54, 10131020, 1992.

Afraimovich, E. L.: Statistical angle-of-arrival and doppler method (SADM) for determining characteristics of the dynamics of the transionospheric radio signal interference pattern, Acta Geod. Geoph. Hung., 32, N3-4, 461-468, 1997.

Afraimovich, E. L., Palamartchouk, K. S., and Perevalova, N. P.: GPS radio interferometry of travelling ionospheric disturbances, J. Atmos. and Sol.-Terr. Phys., 60, N12, 1205-1223, 1998. 
Afraimovich, E. L., Boitman, O. N., Zhovty, E. I., et al.: Dynamics and anisotropy of traveling ionospheric disturbances as deduced from transionospheric sounding data, Radio Sci., 34, N2, 477487, 1999.

Afraimovich, E. L., Kosogorov, E. A., Palamarchouk, K. S., Perevalova, N. P., and Plotnikov, A. V.: The use of GPS arrays in detecting the ionospheric response during rocket launchings, Earth, Planets, and Space, 52, N11, 1061-1066, 2000a.

Afraimovich, E. L.: The GPS global detection of the ionospheric response to solar flares, Radio sci., 35, N6, 1417-1424, 2000b.

Afraimovich,. E. L., Palamartchouk, K. S., and Perevalova, N. P.: Statistical angle-of-arrival and doppler method for GPS interferometry of TIDs, Adv. Space Res., 26, N6, 1001-1004, 2000c.

Afraimovich, E. L., Kosogorov, E. A., Lesyuta, O. S., et al.: Geomagnetic control of the spectrum of traveling ionospheric disturbances based on data from a global GPS network, Ann. Geophysicae, 19, N7, 723-731, 2001a.

Afraimovich, E. L., Kosogorov, E. A., Perevalova, N. P., and Plotnikov A. V.: The use of GPS-arrays in detecting shock-acoustic waves generated during rocket launchings, J. Atmos. and Sol.Terr. Phys., 63, N18, 1941-1957, 2001b.

Afraimovich, E. L., Perevalova, N. P., Plotnikov, A. V. and Uralov A. M.: The shock-acoustic waves generated by earthquakes, Ann. Geophysicae, 19, 395-409, 2001c.

Bertel, L., Bertin, F., and Testud, J.: De la mesure du contenu electronique integre appliquee a l'observation des ondes de gravite de moyenne echelle, J. Atmos. and Sol.-Terr. Phys., 38, 261-270, 1976.

Bowman, G. G.: Quasi-periodic scintillations at mid-latitudes and their possible association with ionospheric E-sporadic structures, Ann. Geophysicae, 7, N3, 259-268, 1989.

Calais, E. and Minster, J. B.: GPS detection of ionospheric perturbations following the January 1994, Northridge earthquake, Geophys. Res. Lett., 22, 1045-1048, 1995.

Calais, E. and Minster, J. B.: GPS detection of ionospheric perturbations following a Space Shuttle ascent, Geophys. Res. Lett., 23, 1897-1900, 1996.

Calais, E., Minster, J. B., Hofton, M. A., and Hedlin, M. A. H.: Ionospheric signature of surface mine blasts from Global Positioning System measurements, Geophys. J. International, 132, 191-202, 1998a.

Calais, E., Minster, J. B., and Bernard, J.: GPS, Earthquake, the ionosphere and Space Shuttle, Physics of Earth and Planet, 105,
167-181, 1998b.

Fitzgerald, T. J.: Observations of total electron content perturbations on GPS signals caused by a ground level explosion, J. Atmos. and Terr. Phys., 59, 829-834, 1997.

Gurtner, W.: RINEX: The Receiver Independent Exchange Format Version 2, http://igscb.jpl.nasa.gov:80/igscb/data/format/rinex2. txt, 1993.

Hocke, K. and Schlegel, K.: A review of atmospheric gravity waves and traveling ionospheric disturbances: 1982-1995, Ann. Geophysicae, 14, 917-940, 1996.

Hofmann-Wellenhof, B., Lichtenegger, H., and Collins, H.: Global Positioning System: Theory and Practice, Springer-Verlag Wien, New York, 327 p., 1992.

Kalikhman A. D.: Medium-scale traveling ionospheric disturbances and thermospheric winds in the F-region, J. Atmos. Sol. Terr Phys., 42, 697-703, 1980.

Karasawa, Y., Yasukawa, K., and Yamada, M.: Ionospheric scintillation measurements at $1.5 \mathrm{GHz}$ in mid-latitude region, Radio Sci., 20, N3, 643-651, 1985.

Klobucar J. A.: Ionospheric time-delay algorithm for singlefrequency GPS users, IEEE Trans. on Aerospace and Electronic system, AES, 23, N3, 325-331, 1986.

Korn, G. A. and Korn, T. M.: Mathematic handbook, 2nd edition, McGraw-Hill Book Company, 1968.

Li, Y. Q., Jacobson, A. R., Carlos, R. C., Massey, R. S., Taranenko, Y. N., and Wu, G.: The blast wave of the Shuttle plume at ionospheric heights., Geophys. Res. Lett., 21, 2737-2740, 1994.

Mannucci, A. J., Ho, C. M., Lindqwister, U. J., Runge, T. F., Wilson, B. D., and Yuan, D. N.: A global mapping technique for GPS-drived ionospheric TEC measurements, Radio Sci., 33, 565-582, 1998.

Mercier, C. and Jacobson, A. R.: Observations of atmospheric gravity waves by radio interferometry: are results biased by the observational technique, Ann. Geophysicae, 15, 430-442, 1997.

Oliver, W. L., Otsuka, Y., Sato, M., Takami, T., and Fukao, S.: A climatology of F-region gravity waves propagation over the middle and upper atmosphere radar, J. Geophys. Res., 102, 14449 $14512,1997$.

Spoelstra, T. A. Th. and Kelder, H.: Effects produced by the ionosphere on radio interferometry, Radio Sci., 19, 779-788, 1984.

Titheridge, J. E.: The diffraction of satellite signals by isolated ionospheric irregularities, J. Atmos. and Terr. Phys., 33, 47-69, 1971 